\title{
Redox Systems in Botrytis cinerea: Impact on Development and Virulence
}

\author{
Anne Viefhues, Jens Heller, Nora Temme, and Paul Tudzynski \\ Institut fuer Biologie und Biotechnologie der Pflanzen, Westfaelische Wilhelms Universitaet Muenster, Schlossplatz 8 , \\ D-48143 Muenster, Germany
}

Submitted 21 January 2014. Accepted 21 March 2014.

The thioredoxin system is of great importance for maintenance of cellular redox homeostasis. Here, we show that it has a severe influence on virulence of Botrytis cinerea, demonstrating that redox processes are important for hostpathogen interactions in this necrotrophic plant pathogen. The thioredoxin system is composed of two enzymes, the thioredoxin and the thioredoxin reductase. We identified two genes encoding for thioredoxins (bctrx1, bctrx2) and one gene encoding for a thioredoxin reductase (bctrrl) in the genome of $B$. cinerea. Knockout mutants of bctrx 1 and bctrr 1 were severely impaired in virulence and more sensitive to oxidative stress. Additionally, $\Delta$ bctrr1 showed enhanced $\mathrm{H}_{2} \mathrm{O}_{2}$ production and retarded growth. To investigate the impact of the second major cellular redox system, glutathione, we generated deletion mutants for two glutathione reductase genes. The effects were only marginal; deletion of bcglr1 resulted in reduced germination and, correspondingly, to retarded infection as well as reduced growth on minimal medium, whereas bcglr2 deletion had no distinctive phenotype. In summary, we showed that the balanced redox status maintained by the thioredoxin system is essential for development and pathogenesis of $B$. cinerea, whereas the second major cellular redox system, the glutathione system, seems to have only minor impact on these processes.

In a permanently changing environment, organisms constantly need to adapt to diverse perturbations. Exposed to an aerobic environment, species evolved aerobic metabolic processes, which unavoidably led to the conversion of oxygen to reactive oxygen species (ROS). ROS are constantly produced as byproducts of several metabolic pathways; therefore, they are located in various cellular components such as the mitochondria, peroxisomes, and chloroplasts. They have diverse physiological roles in organisms and are involved in different cellular processes, e.g., proliferation, differentiation, and signal transduction (Aguirre et al. 2005; Heller and Tudzynski 2011). Their diffusible character allows them to serve as secondary messengers for several signaling pathways (Chen et al. 2009). However,

Current address for J. Heller: Department of Plant and Microbial Biology, University of California Berkeley, Berkeley, CA 94720, U.S.A.

Current address for N. Temme: PLANTA Angewandte Pflanzengenetik und Biotechnologie GmbH Grimsehlstr. 31 D-37555 Einbeck, Germany.

Corresponding author: P. Tudzynski; E-mail: tudzyns@uni-muenster.de

* The $\boldsymbol{e}$-Xtra logo stands for "electronic extra" and indicates that five supplementary figures and two supplementary tables are published online.

(C) 2014 The American Phytopathological Society the exposure of biological molecules to ROS also leads to severe molecular damage, especially lipid peroxidation, denatured proteins, and oxidized DNA bases (Beckmann and Ames 1998; Belozerskaia and Gessler 2007). Imbalance of the equilibrium between production and scavenging of ROS causes oxidative stress that needs to be counteracted by the activation of appropriate signaling pathways. All ROS-producing organisms are able to avoid elevated ROS levels by efficient scavenging systems whose expression is, in most cases, elevated under oxidative stress.

Besides the elimination of deleterious oxidants, active release and production of ROS can be enforced by organisms. In plantpathogen interactions, it is known that ROS are crucial for recognition and defense reactions (Heller and Tudzynski 2011). Under pathogen attack, plants release ROS as a defense strategy as part of the hypersensitive response (HR). This HR is featured by the rapid death of cells at the infection site and is effective to antagonize growth of biotrophs that depend on living plant cells (Mur 2008; Rivas and Thomas 2005). This strategy is less effective against necrotrophic fungal plant pathogens such as Botrytis cinerea, also known as the gray mold fungus (Groves and Loveland 1953; Williamson et al. 2007). As a generalist, this pathogen can infect over 200 plant species, including agronomically important crops like strawberries, grapes, and tomatoes and causes huge economic losses during postharvest storage (Elad and Evenses 1995; Williamson et al. 2007; Vicedo et al. 2006). In addition to triggering an oxidative burst in the course of host infection, $B$. cinerea contributes to programmed plant cell death as part of its infection strategy (Govrin and Levine 2000; Shlezinger et al. 2011), and it even raises the concentration of ROS at the infection site by forming its own ROS (Tenberge et al. 2002; Tudzynski and Kokkelink 2009). This raises the question, however, how can $B$. cinerea cope with these elevated amounts of ROS? The gray mold fungus must guarantee a rapid turnover of ROS to sustain the balance between oxidizing and reducing equivalents, which constitute the intracellular redox state (Jones 2008; Torres and Dangl 2005). Detoxifying enzymes like catalases, peroxidases, and superoxide dismutases are supposed to be necessary to counteract these deleterious ROS in planta. However, functional analysis of the transcription factor Bap1 (Botrytis activator protein 1), which controls expression of most of the classical oxidative stress-responsive (OSR) genes in B. cinerea (Temme and Tudzynski 2009), did not support this theory. This factor is crucial for tolerance against oxidative stress in vitro, but it has no influence on the pathogenicity of the fungus. The Bap1controlled genes are also in the wild type (WT) not induced in the plant tissue, suggesting that either the fungus does not face oxidative stress in planta or that alternative systems are used to ensure redox homoeostasis during infection. Here, we present 
a detailed analysis of the impact of the two major cellular redox systems, thioredoxin and glutathione (GSH).

Thioredoxins are small, ubiquitous oxidoreductases that are evolutionarily highly conserved. The proteins are characterized by a redox-active site, characterized by the presence of two vicinal cysteines (CxxC) (Arner and Holmgren 2000; Holmgren 1989). This active-site motif is not exclusively present in thioredoxins but also in glutaredoxins, protein disulfide isomerases, and DsbA (disulfide bond formation), forming a protein superfamily (Arner and Holmgren 2000; Martin 1995). This domain is essential for the reduction of target proteins and is arranged in a characteristic tertiary structure termed Trx fold. The N-terminal Cys of the catalytic site, exposed on the surface, is in close proximity to the second Cys, allowing perfect access of target substrates (Martin 1995; Meyer et al. 2009). To function as an efficient and rapidly reversible redox system, $\mathrm{NADPH}$, as electron donor, and a thioredoxin reductase are necessary to provide reducing equivalents. Thioredoxin reductases, similar to thioredoxins, are homodimeric oxidoreductases. Each monomer of the flavoprotein contains a FAD prosthetic group, a NADPH binding domain, and the redox-active site disulfide (Holmgren and Bjornstedt 1995; Mustacich and Powis 2000). The reduction of oxidized substrates requires the attack of disulfide bridges by the active-site sulfhydryls of thioredoxin, and this action subsequently leads to the release of free thiols. Oxidized thioredoxin $\left(\operatorname{Trx}-\mathrm{S}_{2}\right)$, in turn, can be reduced $\left[\operatorname{Trx}-(\mathrm{SH})_{2}\right]$ enzymatically by the transfer of electrons from NADPH via FAD to the active site of thioredoxin reductase (Fig. 1) (Brandes et al. 1993; Meyer et al. 2009). The reversible oxidation of catalytical or structural thiols is crucial for the biological function of proteins (Arner and Holmgren 2000).

In general, multiple roles can be assigned to the thioredoxin system within a cell. It is necessary for many biosynthetic enzymes, such as ribonucleotide reductases, for dNTP generation, sulfate reductases, and methionine sulfoxide reductases, for the synthesis of amino acids, and for the reconstitution of damaged proteins (Camier et al. 2007; Gonzalez Porque et al. 1970; Holmgren 1989). On the other hand, it is also an important antioxidative system, preventing protein denaturation or aggregation by distinct thiol exchange reactions (Arner and Holmgren 2000). In Saccharomyces cerevisiae, it was shown that the 2-Cys peroxiredoxin (Tsa1) is an important substrate for the detoxification of ROS. Under high $\mathrm{H}_{2} \mathrm{O}_{2}$ concentrations, reduced Tsa1 becomes oxidized at its sulfhydryl group, thereby detoxifying $\mathrm{H}_{2} \mathrm{O}_{2}$. The oxidized sulfhydryl groups of two Tsa1 molecules form disulfide bridges. These homodimers can then be recycled by the thioredoxin system (da Silva Dantas et al. 2010).
Besides the thioredoxin system, the GSH system plays a central role for intracellular redox homeostasis in eukaryotic cells. GSH is one of the key metabolites involved in maintaining a reduced intracellular redox milieu. It is the basic lowmolecular weight thiol in most eukaryotic and prokaryotic cells (Foyer and Noctor 2005). Besides keeping the cellular redox status reduced in general, GSH specifically reduces glutaredoxins, which are enzymes that are structurally similar to thioredoxins. But in contrast to thioredoxins, glutaredoxins are reduced nonenzymatically by GSH, using electrons donated from NADPH. In the course of the reaction, GSH itself is oxidized to GSH disulfide (GSSG). Changes in the redox equilibrium of GSH and GSSG can reversibly modify redox-sensitive thiol groups of target proteins activating various cellular processes (Meyer 2008). To restore the cellular GSH pool in oxidizing conditions, GSSG is constantly reduced with electrons delivered from NADPH in a reaction catalyzed by GSH reductase (Meyer 2008; Toledano et al. 2013). Therefore, GSH reductase is the key enzyme for keeping the GSH pool in a reduced state and, thereby, functional.

As outlined above, the specific infection strategy of B. cinerea indicates that efficient ROS detoxification systems must be active in this fungus to handle overall enhanced ROS levels, due to the induced plant response. However, the results obtained with the major oxidative stress responsive transcription factor Bap1 had shown that the basic enzymatic ROS detoxification systems do not seem to be essential for infection, leading to the assumption that $B$. cinerea does not suffer under oxidative stress in planta. Here, we present evidence that maintenance of the redox status is indeed important for development and virulence of $B$. cinerea and that the thioredoxin system but not the GSH pool is a central player in these processes.

\section{RESULTS}

\section{Identification and characterization of BcTrx1, BcTrx2 and BcTrr1.}

The open reading frames (ORF) encoding two $B$. cinerea thioredoxins (BcTrx1, BcTrx2) and the thioredoxin reductase BcTrr1 are annotated in the genomic database of the Broad Institute. Sequence analysis of polymerase chain reaction (PCR) and reverse transcription-PCR products confirmed the coding sequence of the respective genes (Table 1.). Both thioredoxins contain the characteristic active-site motif $\mathrm{CxxC}$, the thioredoxin domain, which can catalyze redox reactions, and clearly belong to the oxidoreductase family. The thioredoxin reductase BcTrr1 is another member of this family and contains the characteristic oxidoreductase domain, which is part of the redoxactive site, as well as NAD- and FAD-binding domains for the

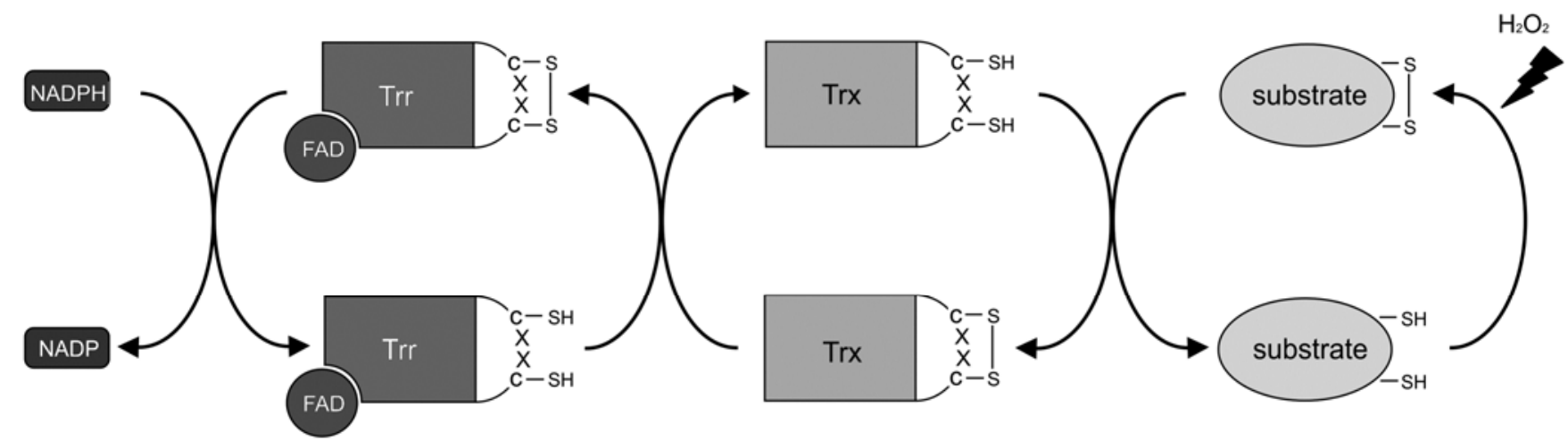

Fig. 1. Reduction of oxidized substrates by the thioredoxin system. Upon oxidative stress, oxidized substrates can be recycled by the thioredoxin system. The reducing power of thioredoxin (Trx) allows the attack of disulfide bridges in target proteins and the release of reduced substrates. The thioredoxin reductase (Trr) uses electrons of NADPH to catalyze the conversion of oxidized $\left(-\mathrm{S}_{2}\right)$ to reduced $\left[(\mathrm{SH})_{2}\right]$ thioredoxin. 
reduction of oxidized thioredoxin. Protein alignments of BcTrr1 and BcTrx1 with other protein primary structures revealed a significant number of residues identical to those of other described thioredoxins and thioredoxin reductases, respectively, particularly in the catalytic core (Supplementary Fig. S1). Classification of additional thioredoxin genes proved to be more difficult, because many homologous thioredoxin-like proteins could be identified. A phylogenetic comparison was conducted using the program Phylogeny.fr (Information Génomique et Structurale, Marseille, France) as well as sequence alignment of the core sequences. We identified B0510_928 as the best candidate for a putative second trx gene and included it as bctrx 2 in our analyses. To assess the role of the thioredoxin system in redox homeostasis and pathogenicity, deletion mutants were generated. For targeted deletion of the particular genes, the ORF of interest was replaced with a hygromycin and nourseothricin resistance (hyg ${ }^{\mathrm{R}}$ and nat ${ }^{\mathrm{R}}$, respectively) cassette (Supplementary Fig. S2A). Diagnostic PCR confirmed independent homokaryotic transformants for $\Delta$ bctrx1 (T1, T5, T9), $\Delta$ bctrr1 (T6, T14, T15), $\Delta$ bctrx2 (T1, T3, T8), and $\Delta$ bctrx1/2 (T2, T10) (data not shown). Southern blot analyses of the transformants confirmed homologous integration of the replacement fragments in locus in the genome and excluded the possibility of further ectopic integration events. The independent mutants of the respective genes exhibited the same phenotypes. In this study, results of one transformant per construct $(\Delta$ bctrx $1 \mathrm{~T} 5, \Delta$ bctrr1 T15, $\Delta$ bctrx $2 \mathrm{~T} 3$, and $\Delta$ bctrx $1 / 2$ T2) are presented. Complementation vectors were transformed into the respective deletion mutants and integration was proved by diagnostic PCR (Supplementary Fig. S5A). Pathogenicity tests as well as growth and germination assays confirmed that the WT phenotype was restored.

\section{The thioredoxin system is crucial for the oxidative stress response.}

To analyze vegetative growth of the mutants, the strains were either grown on complete solid medium (CM) or CM with different supplements (Fig. 2). The morphology of $\Delta$ bctrr1 and the bctrx 1 and bctrx 2 double deletion ( $\Delta$ bctrx $1 / 2)$ seemed to be disturbed in comparison with the WT, as they showed less than 50 and $65 \%$, respectively, of the radial growth of the WT after incubation for 3 days (Supplementary Fig. S3A). Although the mutants were reduced in growth, they were generally able to sporulate and to form sexual reproduction structures (data not shown). The growth and development of $\Delta$ bctrx 1 and $\Delta$ bctrx2 was WT-like. To investigate the impact of the loss of components of the thioredoxin system on cellular redox balance during the adaptation to environmental changes, growth under oxidative and osmotic stress was analyzed. Osmotic stress, i.e., applying high sugar (glucose) or salt $(\mathrm{NaCl})$ concentrations, did not significantly affect the growth rates of the mutants. Different oxidative stressors had diverse effects on the mutants. Hydrogen peroxide had a strong impact, as growth drastically declined along with increased $\mathrm{H}_{2} \mathrm{O}_{2}$ concentrations, while the influence of menadione was less severe (Fig. 2B). The membrane-active agent sodium dodecyl sulfate (SDS) also seemed to have a negative influence on the growth of $\Delta$ bctrr1, as the mutant only showed $25 \%$ radial growth. The WT and the other tested mutants still had growth rates of about $60 \%$. The cellwall stressor Congo red had no specific effect on either of the mutants (data not shown), in contrast to the $\Delta$ trx mutants in Magnaporthe oryzae (Fernandez and Wilson 2014).

In some fungi, the deletion of components of the thioredoxin system results in auxotrophy for sulfur-containing amino acids due to the defective reduction of the oxidized 3'-phosphoadenylsulfate reductase, an enzyme that is mandatory for inorganic sulfate assimilation (Malagnac et al. 2007; Muller 1991). Growth on minimal medium was therefore tested. Growth of $\Delta$ bctrx $1 / 2$ could be significantly enhanced by the addition of either cysteine or methionine to the Czapek Dox minimal medium (CD). Also, growth rates of $\Delta$ bctrx 1 and $\Delta$ bctrr1 were slightly increased. This result suggests an involvement of the thioredoxin system in the (re-)generation of sulfur-containing amino acids.

\section{The thioredoxin system has a strong impact on pathogenicity.}

Next to the vegetative growth, the development of conidiospores was also analyzed. Microscopic examination revealed no discrepancy in size or shape, but differences in the germination process were evident. Since it has been shown that germination of $B$. cinerea conidia is nutrient-dependent (Doehlemann et al. 2005), droplets of spore suspension in $10 \mathrm{mM}$ glucose were placed on a glass surface; especially $\Delta$ bctrr 1 showed a strong retardation in length and quantity of germinating spores (Fig. 3A and B). After 24 h, only 35\% of the spores had germinated and these differentiated very short germ tubes. $\Delta$ bctrx 1 also was delayed in germination (note sampling at 3 and $6 \mathrm{~h}$ [Fig. 3B]). However, after $12 \mathrm{~h}$, nearly $100 \%$ of the $\Delta$ bctrx 1 spores had germinated (Fig. 3B), but the length of their germlings corresponded only to approximately 10 to $20 \%$ of the length of those of the WT (Fig. 3A). Besides nutrient-dependent germination on glass surfaces, germination was also examined on a hydrophobic surface (polypropylene foil), which is characterized by the formation of short germ tubes. The conidia of both mutants showed normal germination characteristics and no differences from the WT. Quantitative evaluations confirmed this result as the same percentage (about 90\%) of spores of all mutants and the WT generated the expected short germ tubes (data not shown). These data show that the mutants are impaired in nutrient-dependent germination while germination induced by hydrophobic surfaces is normal.

To further examine if the penetration of germlings is also disturbed, the infection of onion epidermal cells was monitored microscopically (Fig. 3C). However, there were no differences observed between the mutants and the WT. All strains formed short germ tubes and the characteristic appressoria-like structures that penetrated the tissue. To study the infection structures at a higher resolution, scanning electron microscopy was performed. Bean leaves were incubated for 16 to $24 \mathrm{~h}$ with conidiospores of the respective mutants and the WT. The analysis revealed that the mutants were generally able to penetrate but, in rare cases, they continued to grow over the epidermis and failed to enter the plant (Fig. 3D). Moreover, the formation of infection cushions based on inoculation with mycelium was analyzed. Infection structures are aggregates of hyphal tips derived from a single highly branched hypha and are assumed to be necessary for penetration (Tenberge 2007). All examined mutants were able to differentiate these structures on onion epidermal cells (Fig. 3C).

Table 1. Details of the analyzed proteins and their coding genes.

\begin{tabular}{|c|c|c|c|c|c|}
\hline Seq_ID & Gene & Genomic sequence & Introns & Amino acids & Protein family \\
\hline B0510_2802 & bctrx 1 & $769 \mathrm{bp}$ & 244 bp, 99 bp & 141 & Oxidoreductase \\
\hline B0510_928 & bctrx 2 & $735 \mathrm{bp}$ & $53 \mathrm{bp}, 52 \mathrm{bp}$ & 209 & Oxidoreductase \\
\hline B0510_1511 & bctrrl & $1,390 \mathrm{bp}$ & 58 bp, 75 bp, 77 bp, 139 bp & 347 & Oxidoreductase \\
\hline
\end{tabular}



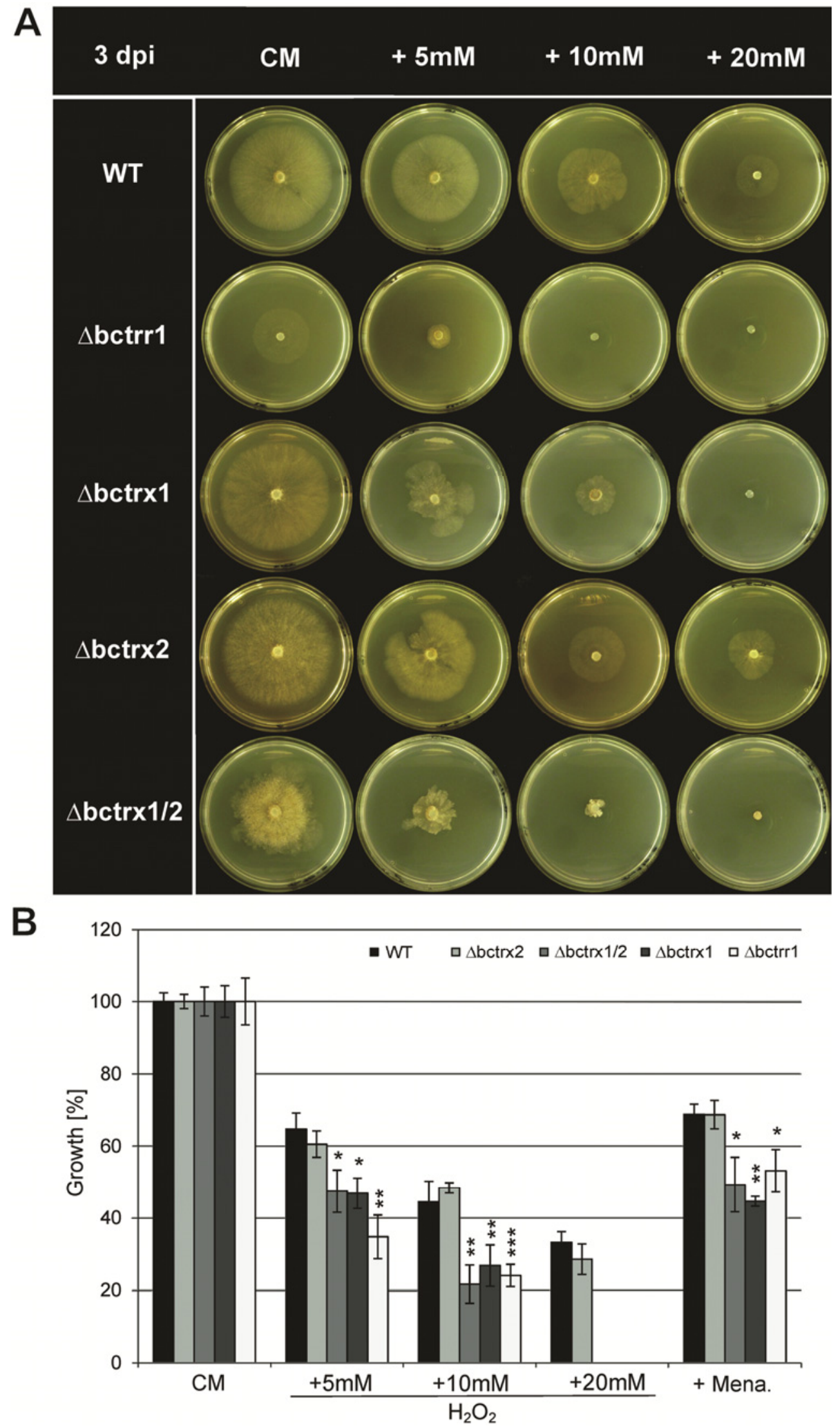

Fig. 2. Growth of $\Delta b c t r x 1, \Delta b c t r x 2, \Delta b c t r x 1 / 2$, and $\Delta$ bctrr 1 under oxidative stress conditions compared with wild-type B05.10. Strains were grown for 3 days on complete medium (CM) supplemented with 5, 10, or $20 \mathrm{mM} \mathrm{H}_{2} \mathrm{O}_{2}$ and $500 \mu \mathrm{M}$ menadione. A, Influence of $\mathrm{H}_{2} \mathrm{O}_{2}$ on fungal growth. Pictures were taken after 3 days. B, Radial growth rates under oxidative stress conditions. Colony diameters were measured at 3 days postinoculation. Growth of each strain on CM medium (without stressing agent) was set to $100 \%$ and relative growth is shown. Mean values and standard deviations were calculated for three colonies per strain and condition. Three independent experiments were performed. Asterisks represent significant differences ( $t$-test) compared with the wild type (WT) in each condition $(*=P \leq 0.05 ; * *=P \leq 0.01 ; * * *=P \leq 0.001)$. 
To address the question whether the thioredoxin system plays a role in pathogenesis, an infection assay was done on primary leaves of Phaseolus vulgaris (Fig. 4A). The leaves were inoculated with a conidial suspension (details below) and were incubated up to 7 days. The $\Delta$ bctrx $1, \Delta$ bctrx $1 / 2$, and $\Delta$ bctrr 1 mutants were severely disturbed in pathogenicity, as only primary lesions were visible even at $7 \mathrm{dpi}$ (days postinoculation). Longer incubation did not lead to further spreading of the lesions. Occasionally, the leaves showed chlorosis but, in most cases, even secondary leaves of the plant were formed normally. In contrast, the WT and the $\Delta$ bctrx 2 mutant formed primary spreading lesions at $2 \mathrm{dpi}$ and, finally, macerated the whole plant tissue. At 7 dpi, new conidia were formed. As wounding of the bean plants did not significantly enhance lesion formation (Fig. 4C), defects in penetration do not seem to be the main reason for the disturbance in virulence. To test if the retardation in infection of the $\Delta$ bctrx 1 and $\Delta$ bctrrl mutants was a host-specific effect, other typical hosts of $B$. cinerea were analyzed. On apple and wounded tomato, the lesion development of $\Delta$ bctrx 1 was delayed in comparison with the WT, but $\Delta$ bctrr1 was still almost incapable of forming lesions on these host tissues (Fig. 4B). To exclude the possibility that defects in pathogenicity are caused during germination, mycelial plugs were used for an infection assay on bean leaves. The results obtained showed a rather irregular infection and, for $\Delta$ bctrx 1 , reduced lesion size of more than $50 \%$ at 2 dpi but $\Delta$ bctrr 1 was
A

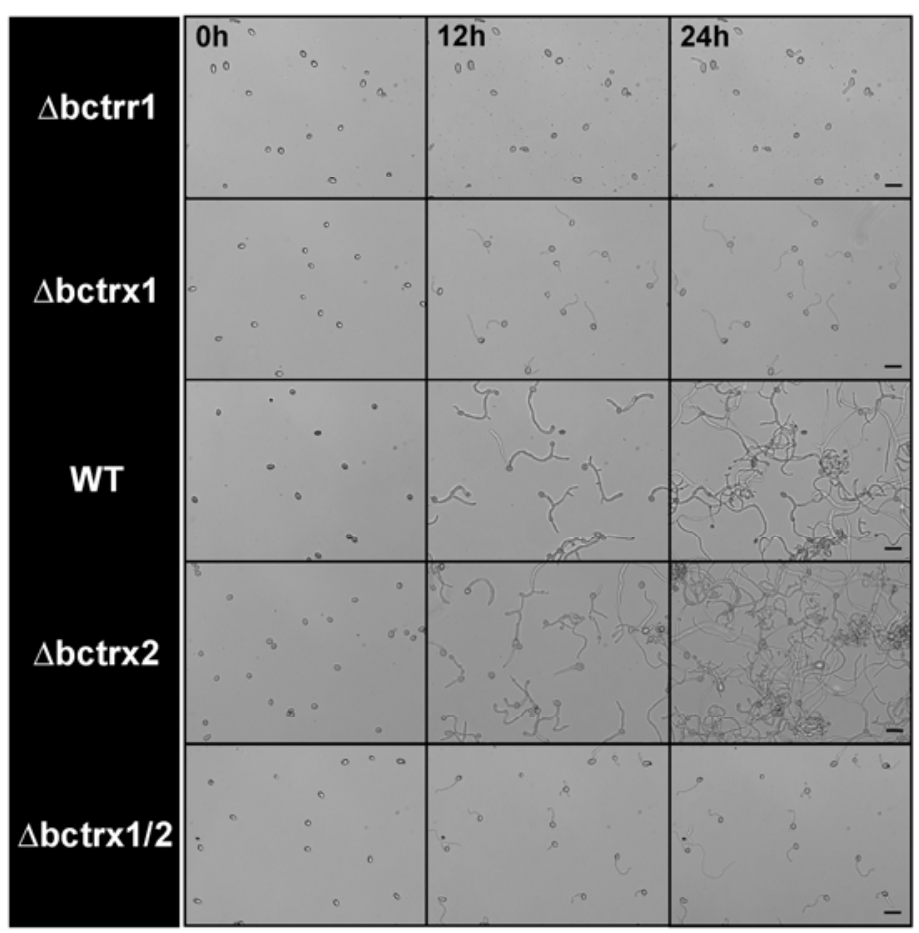

B

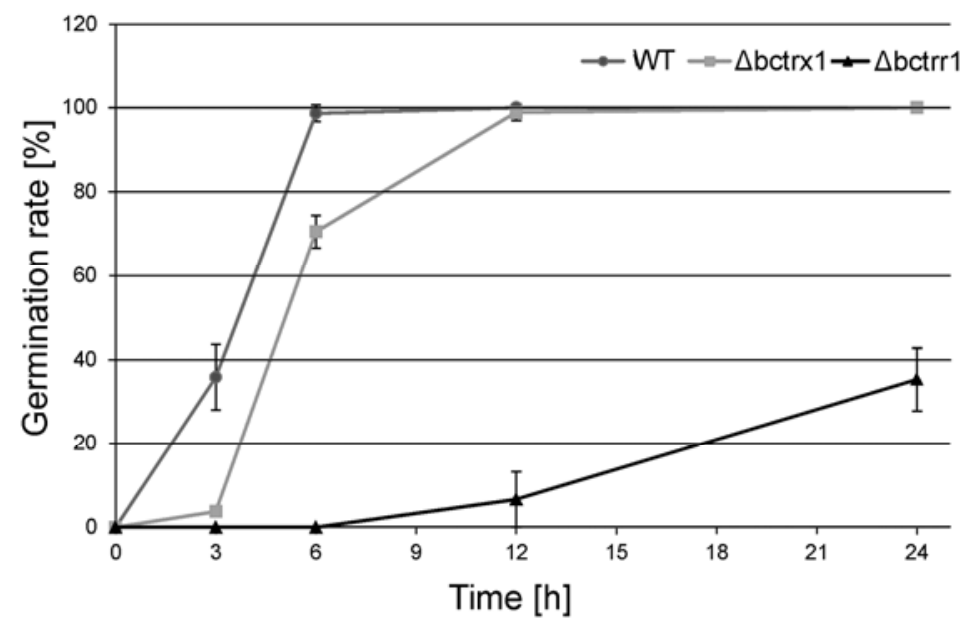

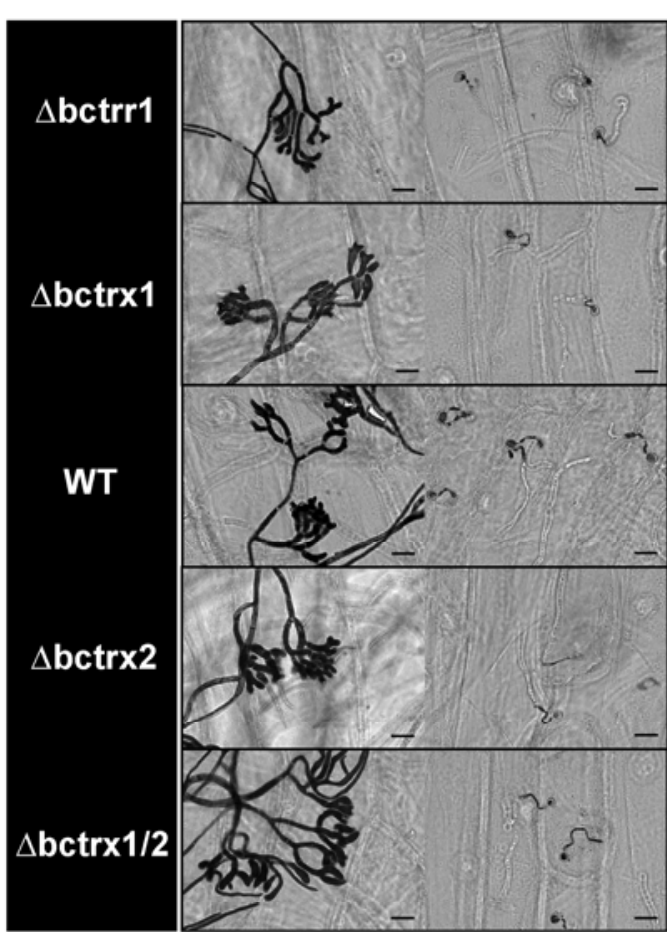
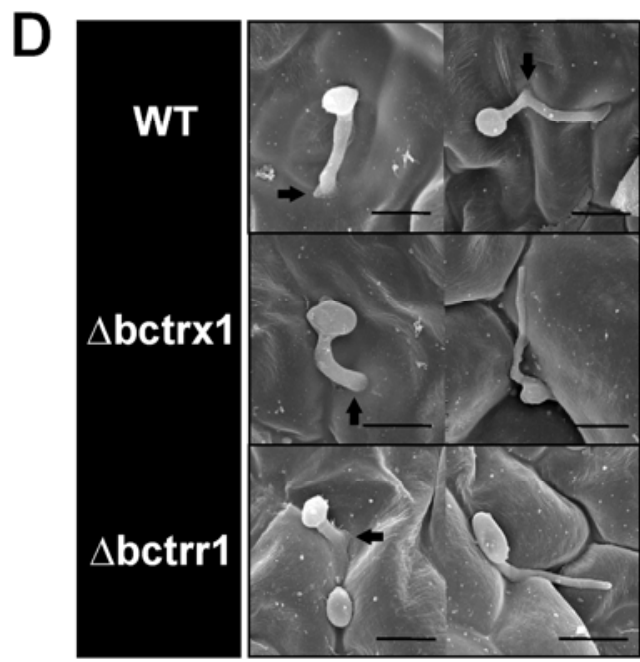

Fig. 3. Evaluation of conidial development and differentiation of infection structures. A, Time course experiment to monitor sugar-induced conidial germination. Conidia were prepared in GB5 $+10 \mathrm{mM}$ glucose and dropped on a glass slide. Scale bar $=20 \mu \mathrm{m}$. B, Germination rates of $\Delta \mathrm{bctrx} 1, \Delta \mathrm{bctrr} 1$, and wild type (WT) conidia in GB5 + $10 \mathrm{mM}$ glucose on a glass surface. Represented are the mean values and standard deviations of two independent experiments with 100 conidia per strain. C, Visualization of infection structures. Onion epidermis was inoculated with mycelia (left) and conidia (right) and was incubated for $24 \mathrm{~h}$. Lactophenol blue was used to stain conidia and hyphae on the surface, while invading hyphae remain colorless. Scale bar $=20 \mu \mathrm{m}$. D, Scanning electron microscopy (SEM) images of Botrytis cinerea-infected bean leaves. Conidia suspension $\left(2 \times 10^{6}\right.$ spores per milliliter $)$ of $\Delta$ bctrx $1, \Delta \mathrm{bctrr} 1$, and WT were dropped on detached bean leaves and were incubated for $18 \mathrm{~h}$. The samples were prepared for SEM by glutaraldehyde fixation, ethanol series, critical point drying, and gold sputter-coating. Arrows indicate appressoria-like structures. Scale bars $=15 \mu \mathrm{m}$. 


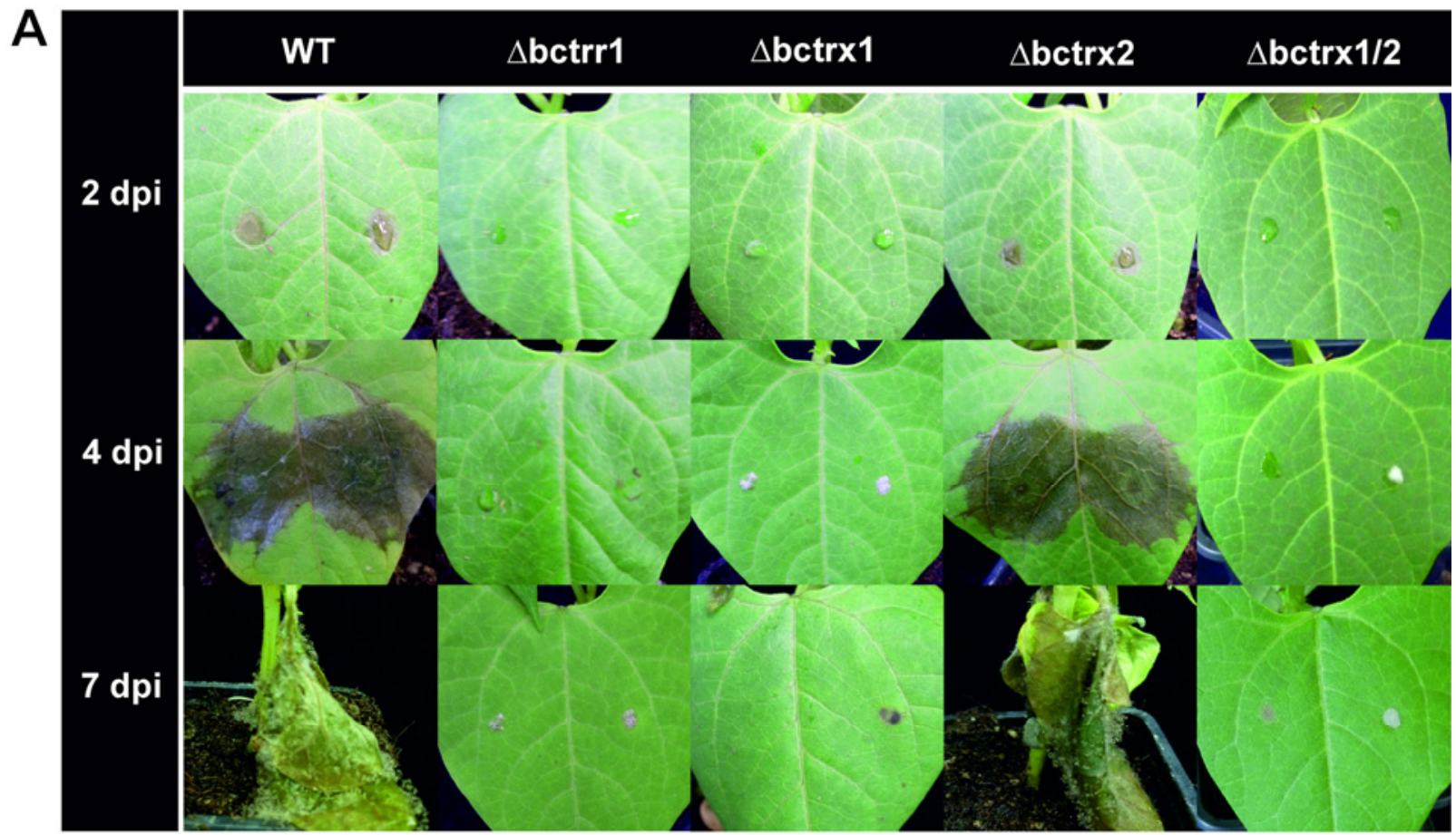

B
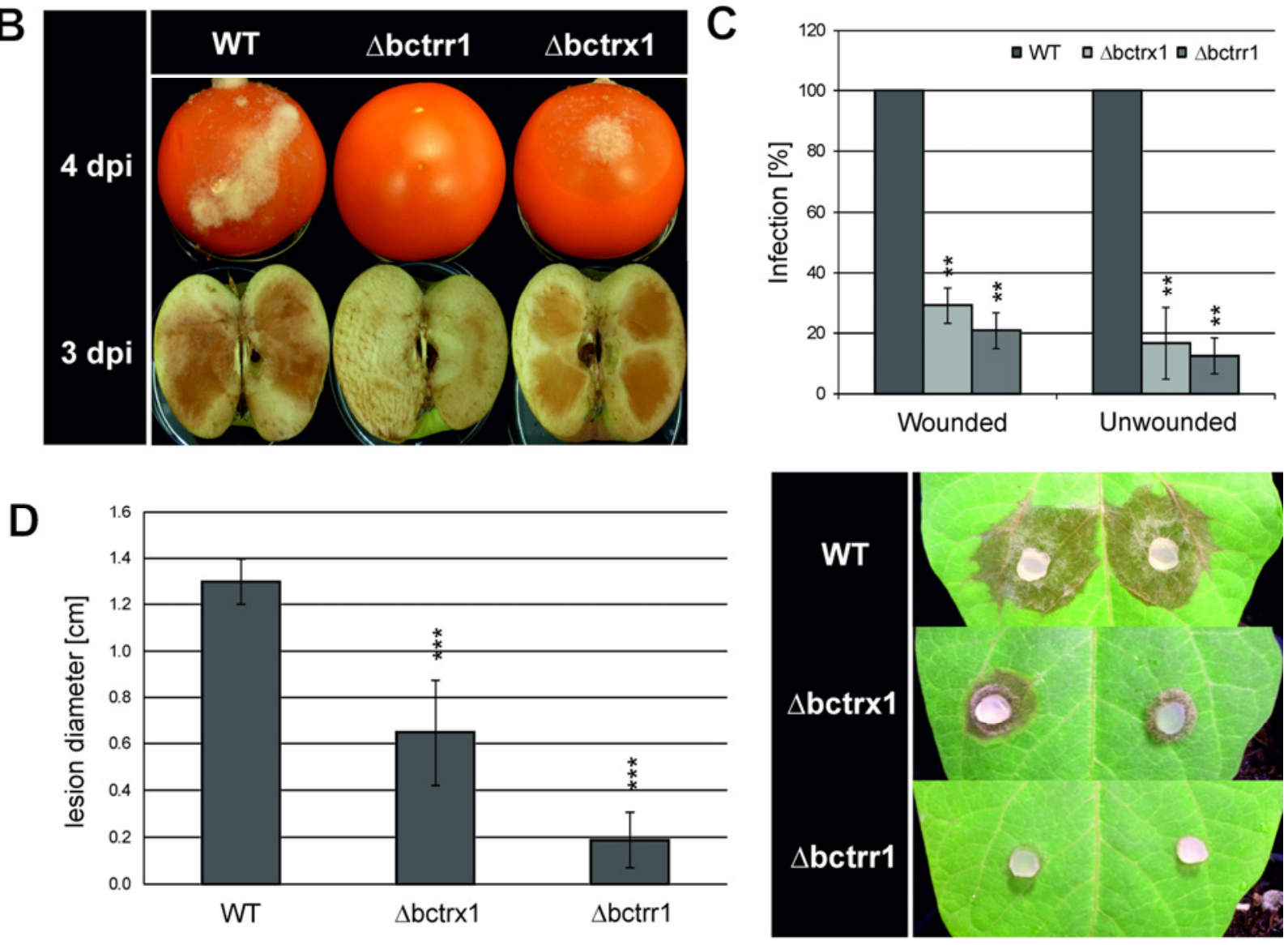

Fig. 4. Pathogenicity assays with mutants of the thioredoxin system. A, Infection of primary bean leaves of Phaseolis vulgaris. Leaves were inoculated with droplets of conidial suspension and were incubated for 7 days. B, Infection of detached tomato fruits and apples with droplets of conidial suspension of $\Delta$ bctrx1 and $\Delta$ bctrr1 in comparison with the wild type (WT). Apples were cut and tomatoes were wounded prior to infection. The infection was monitored for 7 days. Pictures were taken at 3 and 4 days postinoculation (dpi), respectively. C, Measurement of lesion diameter of indicated mutants after inoculation of wounded and unwounded bean leaves. Shown are the mean diameters and standard deviations of two independent experiments with 24 infections per strain and condition. Asterisks represent significant differences compared with the WT in each condition $(*=P \leq 0.05 ; * *=P \leq 0.01 ; * * *=P \leq 0.001)$. D, Plant infection with nonsporulating mycelia and measurement of resulting lesion diameters at 2 dpi. Shown are the mean values and respective standard deviations. In two independent experiments, 24 infections per strain were analyzed. Asterisks represent significant differences ( $t$-test) compared with the WT in each condition $(*=P \leq 0.05 ; * *=P \leq 0.01 ; * * *=P \leq 0.001)$. 

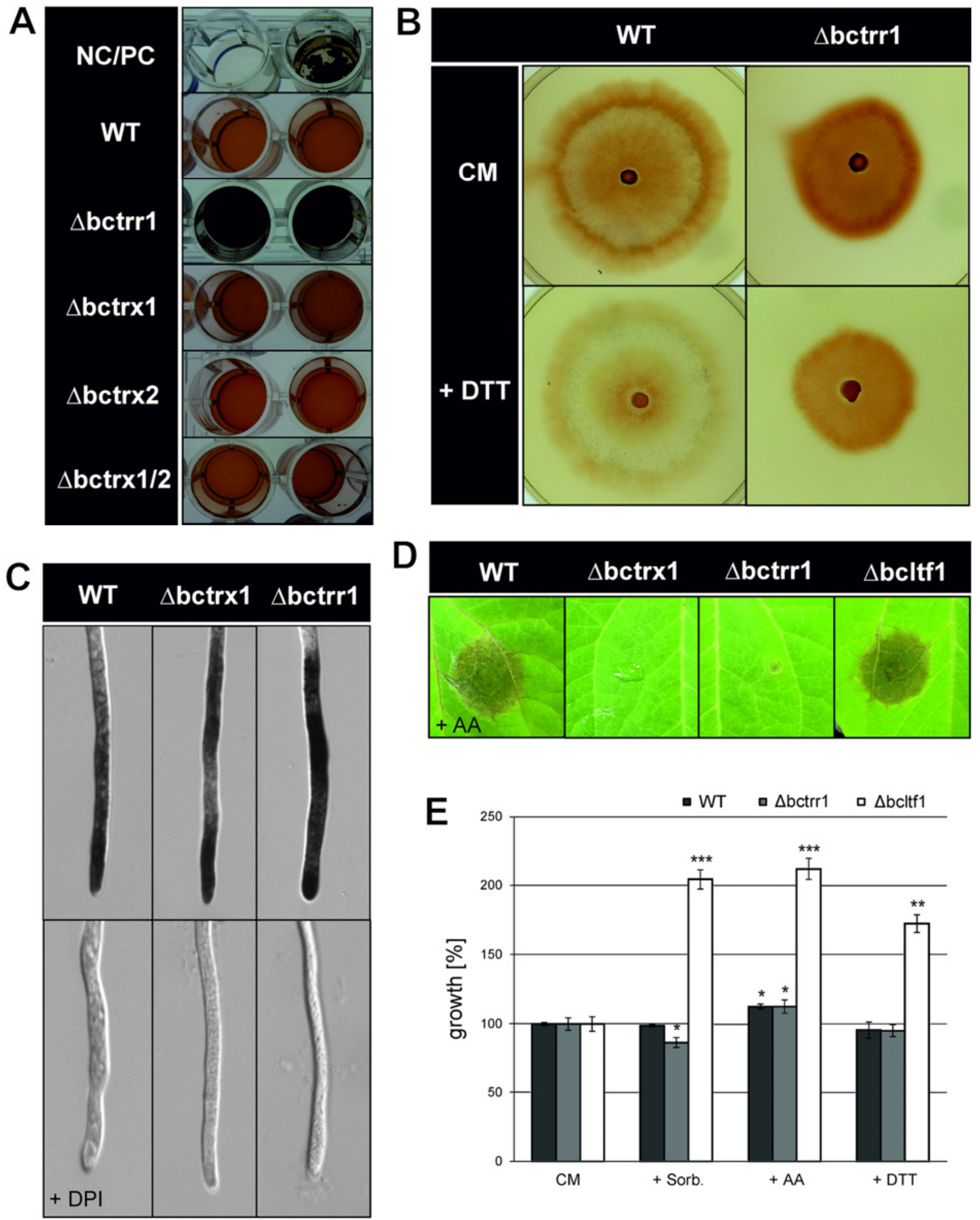

Fig. 5. Reactive oxygen species production and influence of antioxidants. A, Diaminobenzidine (DAB) test for the analysis of $\mathrm{H}_{2} \mathrm{O}_{2}$ production of indicated mutants compared with the wild type. Fresh mycelium was weighed and $1 \mathrm{ml}$ of DAB solution was added. Incubation took place for $1.5 \mathrm{~h}$ in the dark. $\mathrm{NC}=$ negative control, DAB solution $+1 \mu \mathrm{H}_{2} \mathrm{O}_{2} ; \mathrm{PC}=$ positive control, DAB solution $+1 \mu_{1} \mathrm{H}_{2} \mathrm{O}_{2}+1 \mu$ horseradish peroxidase. Three independent experiments in triplicate per strain were performed. B, DAB assay on solid medium. The strains were grown for 2 days on complete medium $(\mathrm{CM})$ or $\mathrm{CM}+750 \mu \mathrm{M}$ dithiothreitol (DTT). The plates were flooded with DAB solution and were incubated for $2 \mathrm{~h}$ in darkness. Images were taken the following day. $\mathbf{C}$, Superoxide detection of $\Delta \mathrm{bctrx} 1, \Delta \mathrm{bctrr} 1$, and WT via nitro blue tetrazolium (NBT); $0.05 \%$ (wt/vol) NBT aqueous was added to the conidial suspensions $\left(1 \times 10^{5}\right.$ spores per milliliter). As a control, $50 \mu \mathrm{M}$ diphenyleneiodonium (DPI) was added prior to supplementation of NBT. The staining was monitored by light microscopy. D, In planta infection assay with ascorbic acid of $\Delta$ bctrx $1, \Delta$ bctrr $1, \Delta$ bcltf 1 , and the WT. Conidia suspensions $\left(2 \times 10^{5}\right.$ spores per milliliter $)$ were supplemented with $5 \mathrm{~g}$ of ascorbic acid per liter prior to inoculation. The pictures were taken at 3 days postinoculation. E, Growth assay of $\Delta \mathrm{bctrr} 1, \Delta \mathrm{bcltf} 1$, and WT under addition of sorbitol, DTT, and ascorbic acid, respectively. CM was supplemented with $1 \mathrm{M}$ sorbitol, $750 \mu \mathrm{M}$ DTT, and $5 \mathrm{~g}$ of ascorbic acid per liter. The plates were incubated for 3 days under light and dark conditions. Given are the mean values and standard deviations resulting from two independent experiments with five plates per strain and condition. Asterisks indicate significant differences $(t$-test) compared with the growth on CM for each condition and strain $(*=P \leq 0.05 ; * *=P \leq 0.01 ; * * *=P \leq 0.001)$. 
almost incapable of infection (Fig. 4D). These studies indicate that the retarded germination is not the (only) reason for the negative impact on pathogenicity.

Summarizing the observed results, the thioredoxin system has a strong impact on pathogenicity. However, a general deficiency in penetration is not the reason for this dysfunction. In contrast, the bctrrl deletion mutant had defective germination, but this phenotype does not explain the retardation in infection, because inoculation with mycelium did not restore pathogenicity.

\section{BcTrr1 controls ROS production and affects oxidative stress response genes.}

$B$. cinerea actively forms ROS in the course of infection. To examine whether this production is disturbed due to the loss of the main intracellular redox system, the production of extracellular $\mathrm{H}_{2} \mathrm{O}_{2}$ was analyzed using the diaminobenzidine (DAB) test, which indicates the secretion of $\mathrm{H}_{2} \mathrm{O}_{2}$ by the formation of a dark brown precipitate. While ROS levels of $\Delta$ bctrx $1, \Delta$ bctrx 2 , and $\Delta$ bctrx $1 / 2$ were comparable to the WT, it was apparent that $\Delta$ bctrr 1 showed strongly increased $\mathrm{H}_{2} \mathrm{O}_{2}$ production (Fig. 5A). To get a closer look at this phenotype, $\Delta$ bctrrl and the WT were grown on CM and CM + dithiothreitol (DTT) agar plates and were stained with DAB. Even in the reducing environment of DTT, $\Delta$ bctrr1 showed a rather dark coloration in comparison with the hardly stained WT mycelium (Fig. 5B). Beside hydrogen peroxide, the superoxide production of $\Delta$ bctrr 1 , detected by nitro blue tetrazolium (NBT) staining, was also enhanced. Staining was predomi- nantly observed in conidia and hyphal tips (Fig. 5C). $\Delta$ bctrr1 showed a stronger accumulation of the black precipitate than did the WT and $\Delta$ bctrx 1 , indicating an increased superoxide production. Diphenyleneiodonium (DPI) acts as an inhibitor of superoxide production by inhibiting flavocytochromes such as, for example, NADPH oxidases. The addition of DPI blocked staining, suggesting that superoxides are generated enzymatically by flavoenzymes. To examine whether the inappropriate ROS production of the bctrrl mutant is responsible for its inability to infect, an antioxidant was added in another pathogenicity test. This experimental approach is based on results obtained with $\Delta$ bcltf1, a mutant lacking a lightresponsive GATA transcription factor, which is also highly sensitive to $\mathrm{H}_{2} \mathrm{O}_{2}$ and produces more $\mathrm{H}_{2} \mathrm{O}_{2}$. In $\Delta$ bcltf 1 , the addition of antioxidants could restore growth defects on solid medium as well as deficiencies in the infection process (Schumacher et al. 2014). It was found that when the antioxidant ascorbic acid was added to the spore suspension prior to inoculation, the treatment could not restore the pathogenicity defect of $\Delta$ bctrr1 (Fig. 5D). Moreover, we attempted to enhance the growth of $\Delta$ bctrr1 on solid medium by adding DTT and ascorbic acid because of their reducing properties and sorbitol as osmotic stabilizer. The addition of ascorbic acid led to a slight increase of growth, however, to the same degree as for the bctrrl mutant and the WT. Sorbitol even reduced the growth of $\Delta$ bctrr1, while DTT had no influence on the growth rate of the WT and the mutants (Fig. 5E).

Since it could be shown that the thioredoxin system was essential for the tolerance of oxidative stresses such as $\mathrm{H}_{2} \mathrm{O}_{2}$,
Peroxiredoxin 2D

Glutaredoxin BcGR1

Glut. Peroxidase BcGpx3

Glutathione reductase BcGIr1

Catalase BcCat2

Stress Protein Ddr48

Heat Shock Protein 12

Hypothetical Protein

Hypothetical Protein

Opsin Bop1
BC1G_09932.1

BC1G_05109.1

BC1G_02031.1

BC1G_15101.1

BC1G_12856.1
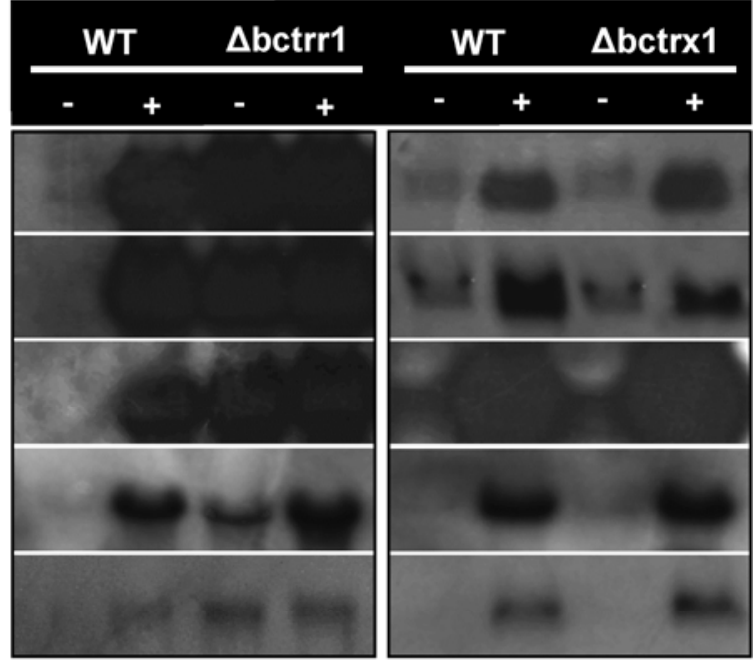

BC1G_10423.1
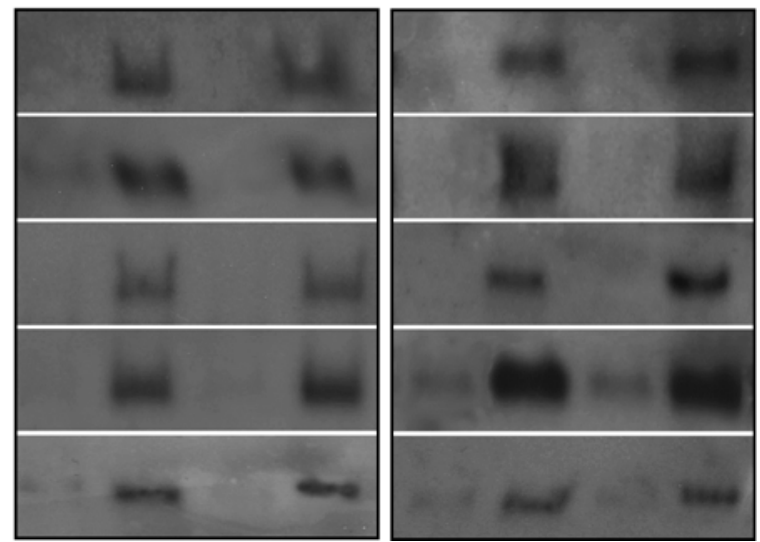

Fig. 6. Effect of bctrrl and bctrxl deletion on gene expression of general and oxidative stress genes. Northern blot analysis of stress response gene expression. Strains were cultivated in liquid Czapek Dox minimal medium supplemented with $10 \mathrm{mM} \mathrm{H}_{2} \mathrm{O}_{2}(+)$ or without $\mathrm{H}_{2} \mathrm{O}_{2}(-)$ for 30 min. 
the transcriptional activation of several OSR genes was evaluated. Northern analyses with $\Delta$ bctrx 1 and $\Delta$ bctrr 1 were performed. The strains were grown in axenic culture supplemented with $10 \mathrm{mM} \mathrm{H}_{2} \mathrm{O}_{2}$ for 30 min. The genes tested encode proteins that are probably directly involved in the oxidative stress response (catalase 2, GSH-peroxidase, glutaredoxin, peroxiredoxin) or in the general stress response (Ddr48, heat shock protein 12, Opsin 1 [Bop1], hypothetical proteins BC1G_12228 and BC1G_15070 [Fig.6]). The results showed a constitutive expression of OSR genes in $\Delta$ bctrrl, while the genes in $\Delta$ bctrx 1 displayed a WT-like expression pattern. Moreover, the general stress response genes are not differentially expressed compared with the WT.
BcTrx1 and BcTrr1 are distributed in the cytosol, while BcTrx2 is localized in the endoplasmic reticulum (ER).

To determine whether the components of the thioredoxin system in $B$. cinerea are restricted to distinct intracellular components, studies with epifluorescence microscopy were performed. The genes of the thioredoxin system were fused to the coding sequence of green fluorescent protein (GFP) under the control of the constitutive promoter oliC from A. nidulans. Microscopic analyses revealed that BcTrx1 and BcTrr1 are evenly distributed throughout the cytosol. On the contrary, BcTrx 2 is restricted to the ER. Co-staining with the nuclear dye Hoechst 33342 and the ER-Tracker Blue-White DPX confirmed the localization (Fig. 7A through C).
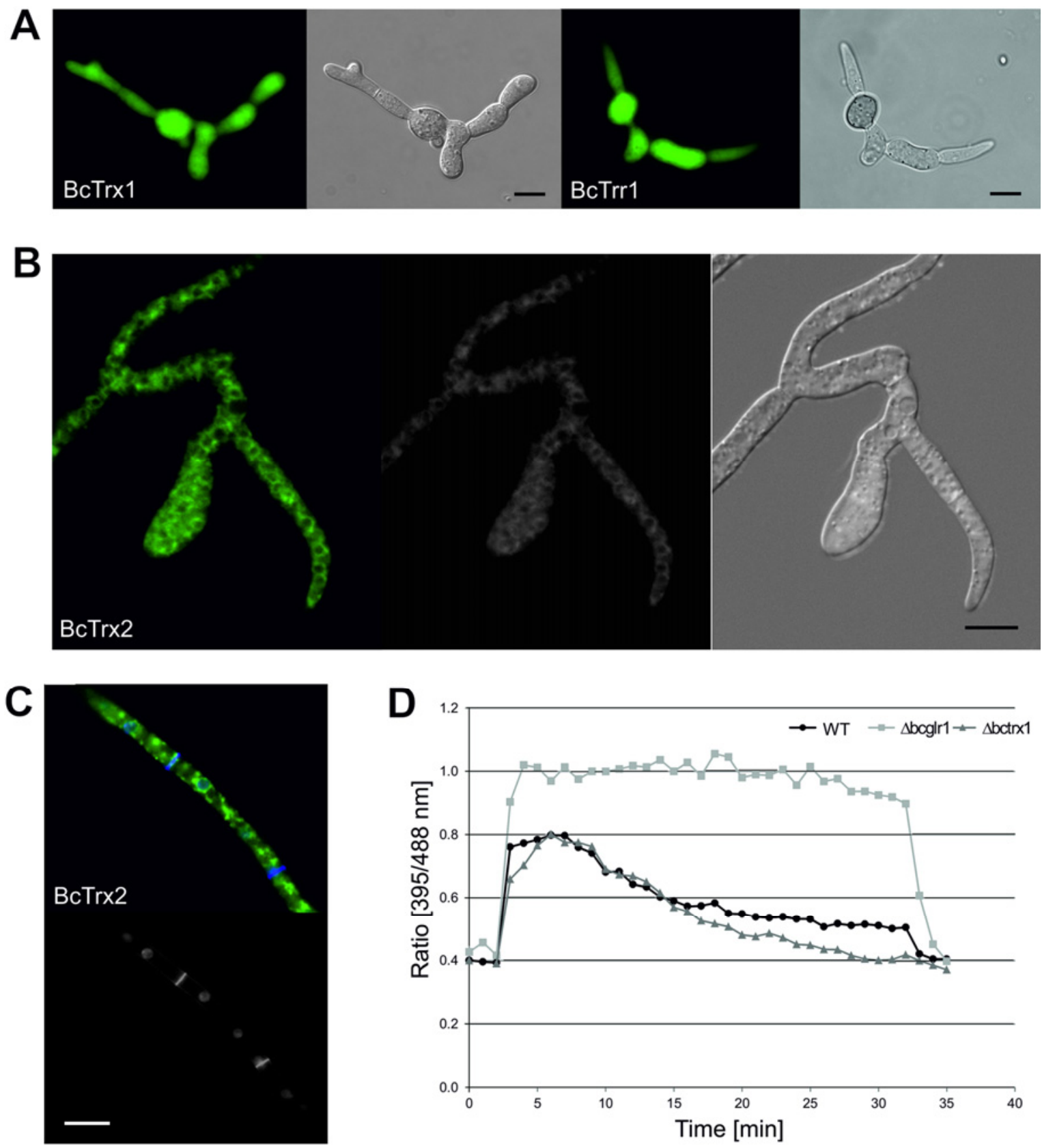

Fig. 7. Cellular localization of BcTrx1, BcTrr1, and BcTrx2 and analysis of the glutathione redox status. A, Overnight-germinated hyphae of bctrx1-gfp and bctrr1-gfp were analyzed via epifluorescence microscopy (left: green fluorescent [GFP], right: bright field). Scale bar $=10 \mu \mathrm{m}$. B and $\mathbf{C}$, BcTrx2 localization by microscopic analysis of bctrx2-gfp germinating hyphae. For co-staining experiments the ER-Tracker Blue-White DPX or Hoechst dye was used. B, From left to right: GFP, ER-tracker, bright field. C, GFP (top), Hoechst (bottom). Scale bar $=10 \mu \mathrm{m}$. D, Redox-dependent changes of grx::rogfp2 fluorescence expressed in different genetic backgrounds ( $\Delta \mathrm{bctrx} 1, \Delta \mathrm{bcglr} 1$, WT). Oxidation was induced using $10 \mathrm{mM} \mathrm{H}_{2} \mathrm{O}_{2}$. For the reduction of redox-sensitive GFP2, $100 \mathrm{mM}$ dithiothreitol was added. Shown are the ratios of fluorescence intensities in the 395 and $488 \mathrm{~nm}$ channels. Represented are the mean values and standard deviations of three different experiments with two repetitions per strain. 
Deletion of bctrx1 has no influence on the GSH pool.

As the thioredoxin system is one of the major redox regulators, does its deletion have an impact on other major redox systems, namely the GSH system? The ratiometric redox-sensitive (ro)GFP was proved to be a suitable tool for in vivo measurements of the redox status of the GSH pool in Botrytis cinerea (Heller et al. 2012). GSH acts as a redox buffer because reduced GSH can be reversibly converted to the oxidized form (GSSG) under oxidizing conditions. Since the GSH reductase constantly reduces GSSG, the redox balance is shifted towards a reduced GSH pool under standard conditions (Meyer et al. 2007). roGFP2 senses the redox potential of the cellular GSH pool and shows different spectral properties depending on its redox state. This sensor was used to analyze the redox milieu of $\Delta$ bctrx 1 in comparison with the WT. Confocal laser scanning microscopy (CLSM) revealed functional $\Delta$ bctrx $1:: G r x-$ roGFP2 transformants as the redox level in single hyphae was changing due to the addition of $\mathrm{H}_{2} \mathrm{O}_{2}$ or DTT (data not shown). To observe differences in the kinetics of GSH after induction of oxidative stress, analyses were performed by fluorometry. Interestingly, the deletion of bctrxl did not cause any differences in GSH recovery after oxidative stress treatment when compared with the WT (Fig.7D). Even repeated induction with $\mathrm{H}_{2} \mathrm{O}_{2}$ and DTT revealed no changes in the GSSG/ GSH curve progression (data not shown). Therefore the general redox status of the cell (using the GSH pool as indicator) seems not to be affected in the $\Delta$ bctrx 1 mutant.

To establish more precisely the role of the GSH system in $B$. cinerea, which seems to act independently of the thioredoxin system, we examined the GSH reductase, the key enzyme responsible for keeping the GSH pool in a reduced state.

\section{Components of the GSH redox system}

have only minor influence on development and virulence.

The GSH reductase bcglr1 (B0510_2036) and bcglr2 (B0510_1402) are annotated in the genomic database of the Broad Institute. The predicted gene lengths (bcglrl, 1,471 bp and bcglr2, 1,851 bp) and the intron-exon structure (Introns, bcglrl $61 \mathrm{bp}$ and bcglr $2319 \mathrm{bp}, 74 \mathrm{bp}$ ) could be confirmed by sequence analyses. The deduced protein BcGlr1 comprises 469 aa and BcGlr2 486 aa. GSH reductases belong to the oxidoreductase family; accordingly putative oxidoreductase domains, including FAD/NAD(P)-binding domains, could be identified by protein structure analysis. Both bcglrl and bcglr2 have been deleted. Diagnostic PCR and Southern blot analysis confirmed homologous integration of the replacement cassette and excluded the possibility of any ectopic integration events (data not shown). For localization experiments, bcglrl-gfp and bcglr2-gfp fusion constructs were synthesized and were also used to complement the deletion strains.

Additionally, $\Delta$ bcglr1::Grx-roGFP mutants were constructed and analyzed by fluorometric measurements, to test if GSH recovery in the mutant is altered. Indeed, the lack of bcglrl resulted in the failure to recover the GSH pool independently after oxidative stress treatment (Fig. 7D). The mutant was able to restore the redox balance of the GSH pool only with the addition of DTT. Therefore the presence of bcglrl is essential for this redox balancing system to function.

To study the virulence of $\Delta$ bcglr 1 and $\Delta$ bcglr2, a bean infection assay was performed as described previously. The infected leaves displayed a normal infection for $\Delta$ bcglr 2 , but a delayed lesion spreading of about 2 days for $\Delta$ bcglr1 (Fig. 8A). However, at $9 \mathrm{dpi}$, the plant was totally macerated indicating an infection process that is merely delayed (data not shown). To prove whether this retardation is due to a defect in spore development, penetration, or surface sensing, germination and penetration assays (onion epidermis) were performed. In comparison

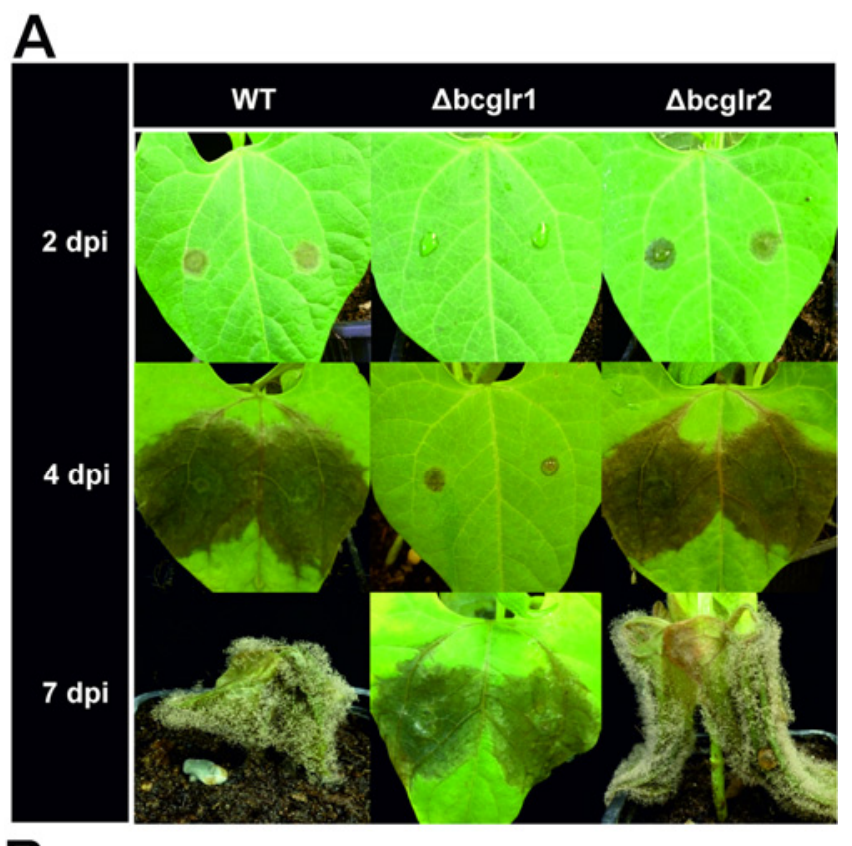

B

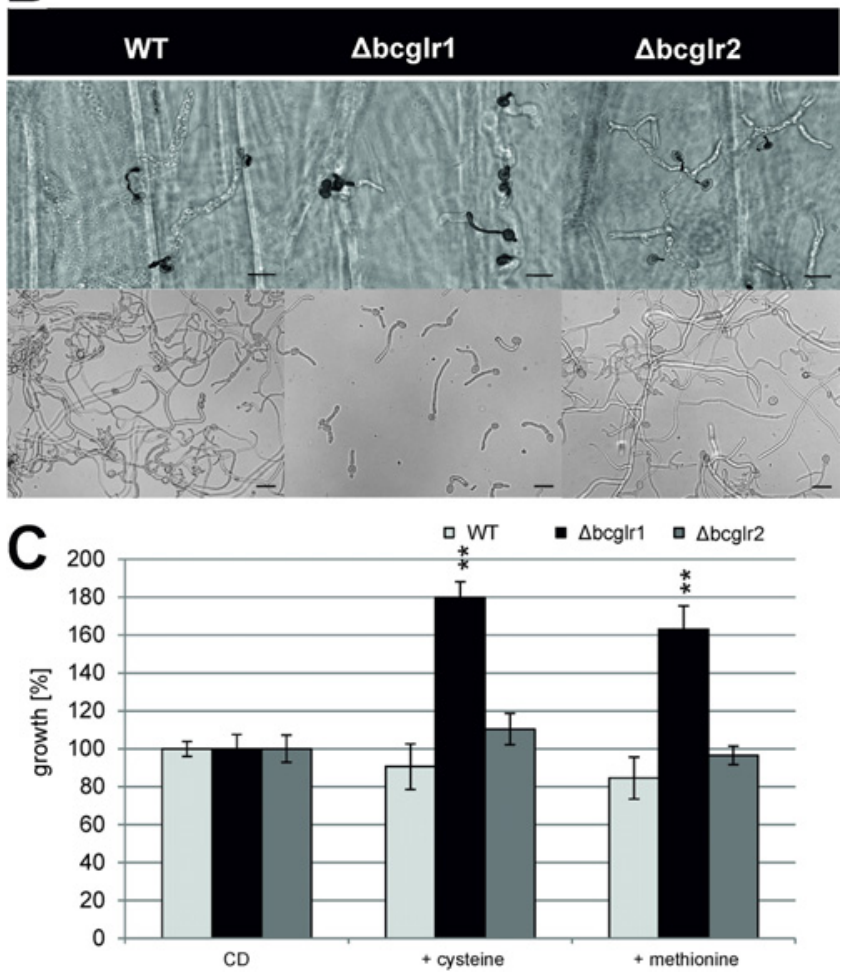

Fig. 8. Virulence and growth assay as well as evaluation of germination and penetration abilities of $\Delta$ bcglr1 and $\Delta$ bcglr2. A, Infection of primary bean leaves of Phaseolis vulgaris with conidia suspension of $\Delta$ bcglr1 and $\Delta$ bcglr2 in comparison with the wild type (WT). Progression of infection was documented at 2, 4, and 7 days postinoculation (dpi). B, Examination of penetration (top) and germination (bottom) of $\Delta$ bcglr1, $\Delta$ bcglr2, and WT spores after $24 \mathrm{~h}$. For analysis of infection structures, onion epidermal strips were inoculated with conidia suspension $\left(1 \times 10^{5}\right.$ spores per milliter and $\left.\mathrm{H}_{2} \mathrm{O}\right)$; for germination, conidia $\left(1 \times 10^{5}\right.$ spores per milliliter and GB5 + $10 \mathrm{mM}$ glucose) were dropped on glass slides and allowed to germinate overnight. Scale bar $=20 \mu \mathrm{m}$. C, Growth assay of $\Delta$ bcglr 1 and $\Delta$ bcglr 2 on Czapek Dox minimal medium (CD) in comparison with WT. In addition, $\mathrm{CD}$ was supplemented with $2 \mathrm{mM}$ cysteine and methionine and growth was measured at 2 dpi. The growth rate on CD was set to $100 \%$ and growth on supplemented media was calculated in relation to the standard medium. The experiments were performed three times with three replicates per strain and condition. Significant differences ( $t$-test) of mean values are represented by asterisks $(*=P \leq 0.05 ; * *=P \leq 0.01 ; * * *=P \leq 0.001)$. 
with the WT, $\Delta$ bcglr2 showed normal germination and penetration behavior, whereas the germination of $\Delta$ bcglr1 was slower as compared with $\Delta$ bctrx 1 (Fig. $8 \mathrm{~B}$ ). Also, the spreading within the onion epidermal tissue seemed to be delayed, but the penetration process itself was not affected (Fig. 8B). As the infection of bean plants with mycelium was WT-like for $\Delta$ bcglr1 and $\Delta$ bcglr2 (Supplementary Fig. S4A) the retarded germination of $\Delta$ bcglr1 may be the reason for the delayed lesion formation in the pathogenicity assay.

As the GSH system, like the thioredoxin system, is involved in redox homeostasis, the oxidative stress response of mutants was analyzed in a plate assay. However, in contrast to $\Delta$ bctrr 1 and $\Delta$ bctrx $1, \Delta$ bcglr 1 and $\Delta$ bcglr2 were not sensitive to oxidative stress by $\mathrm{H}_{2} \mathrm{O}_{2}$ or menadione. Furthermore, osmotic or membrane stress had no influence on the radial growth. In contrast, growth on minimal medium was diminished for $\Delta$ bcglr1. The addition of either cysteine or methionine to the medium resulted in enhanced growth rates comparable to the radial growth of the WT (Fig. 8C). When $\mathrm{H}_{2} \mathrm{O}_{2}$ production was tested by performing a DAB assay, the data did not indicate changed $\mathrm{H}_{2} \mathrm{O}_{2}$ production in comparison with the WT. Localization studies of BcGlr1 and BcGlr2 showed that both proteins clearly accumulated in the cytoplasm.

Summarizing these results, the deletion mutant of bcglr2 does not show any differences in comparison with the WT, while the knockout of bcglrl has a negative influence on conidia germination that explains the retarded lesion formation on plant tissue.

\section{DISCUSSION}

As known from extensive studies in yeast and mammals, thioredoxins have multiple regulatory facets; in addition to redox control and stress response, they are also involved in protection or promotion of oxidative protein folding, programmed cell death, and growth (Holmgren 1985; Holmgren and Bjornstedt 1995; Kern et al. 2003; Powis et al. 2000; Ravi et al. 2005). We could confirm a function in stress response and redox control for the thioredoxin complex in B. cinerea. For the first time, we have demonstrated a central role of the thioredoxin system in the virulence of a plant pathogenic fungus.

However, it is still unknown how necrotrophic fungi react to or use enhanced ROS levels during the infection process. All investigations performed so far suggest that detoxification of extracellular ROS is not needed for infection of $B$. cinerea (Rolke et al. 2004; Schouten et al. 2002; Temme and Tudzynski 2009). Important ROS-detoxifying enzymes, such as catalases, were shown to be entirely dispensable for the pathogenicity process (Schouten et al. 2002). However, because there are many different isoenzymes present, some of their functions may be redundant. This hypothesis does not hold true for Bap1, which is known to be one of the major transcription factors in OSR. The deletion of bapl had no effect on the infection process (Temme and Tudzynski 2009). In planta analysis revealed that Bap1-dependent genes, including bctrrl and bctrxl, are not even induced during plant invasion. Nevertheless, the thioredoxin system is involved in pathogenicity, as the deletion of bctrrl and bctrxl had a severe influence on the infection process, since, in most of the experiments, no lesion spreading or tissue maceration could be observed. In rare cases the leaves turned yellow at 10 to $12 \mathrm{dpi}$, but the plant kept growing by differentiating secondary leaves (data not shown). The discoloration of the tissue might be an indicator of an active defense response of the plant by triggering local cell death (van Doorn and Woltering 2004). Shlezinger and associates (2011) postulated that Botrytis cinerea undergoes massive apoptotic-like programmed cell death (PCD) during early host plant invasion.
Their results suggest that plant defense molecules target the PCD machinery to prevent infection and that the fungal antiapoptotic machinery is crucial to cope with this host-induced PCD. Therefore, during invasion, the thioredoxin system may display anti-apoptotic properties (Shlezinger et al. 2011). Disturbance of the fungal anti-apoptotic machinery would facilitate an efficient plant defense and reduce fungal infection.

$\Delta$ bctrx 1 and $\Delta$ bctrr1 are highly sensitive to ROS, so the oxidative burst of the plant that $B$. cinerea normally exploits might, in this case, be an effective weapon to prevent fungal expansion. Presumably, it causes a complete disturbance of the fungal redox balance that cannot be stabilized due to the absent components of the thioredoxin system. It would be interesting to analyze whether in planta downregulation of genes, e.g., those encoding components of the GSH system or catalases and superoxide dismutases, is the reason for the inability of the mutants to properly infect the host tissue. As there can be significant differences between expression patterns in axenic cultures and in planta, quantitative real time PCR would be a suitable tool to detect in planta expression during pathogenesis. Regarding the pathogenicity effect, this work suggests that a penetration defect can be excluded because onion epidermis infection and scanning electron microscopy (SEM) analyses of infected bean leaves showed normal penetration by both bctrxl and bctrrl mutants (Fig. 3).

The available data, so far, that could establish an influence of the thioredoxin system on fungal pathogenicity are very limited. In the human pathogenic fungus A. fumigatus, inhibition of Trx1 results in sensitivity to oxidative stress and neutrophil-mediated killing in vitro (Leal et al. 2012). However, much is known about the involvement of thioredoxins in bacterial diseases and cancer development in mammals and humans. For example it was shown that, for Salmonella typhimurium $\operatorname{tr} x 1$, deficiency attenuated virulence and that high $\operatorname{tr} x 1$ expression in Helicobacter pylori could be associated with gastric carcinogenesis (Bjur et al. 2006, Shi et al. 2013). In mammals, thioredoxins are pivotal multifunctional enzymes involved in growth promotion, neuroprotection, inflammatory modulation, immune function, and anti-apoptosis, making the thioredoxin system an appealing target for therapeutic development (Mahmood et al. 2013).

As one of the main intracellular redox systems, the influence of oxidative stress on the thioredoxin system could be foreseen. Growth assays indicated a drastic effect of bctrxl and bctrrl deletion on $\mathrm{H}_{2} \mathrm{O}_{2}$ tolerance (Fig. 2A). The effect of supplemented menadione was less severe but significant. Therefore, there is not only a response to hydrogen peroxide but, also, to other ROS, such as the superoxides. In other fungi, isoenzymes often compensate the loss of either thioredoxin or thioredoxin reductase and ensure the viability of the mutants. In Saccharomyces cervisiae, the sctrx 2 mutant shows sensitivity to $\mathrm{H}_{2} \mathrm{O}_{2}$, while the $\Delta$ sctrx 1 mutant does not (Garrido and Grant 2002), indicating that sctrxl deletion is redundant. However, peroxide sensitivity itself is not a ubiquitous phenomenon of the thioredoxin system. While, in Candida albicans, deletion of the major thioredoxin catrxl results in severe sensitivity to $\mathrm{H}_{2} \mathrm{O}_{2}$, in Podospora anserina, deletion of either patrx1, patrx2, or patrx3 has no effect on peroxide tolerance (da Silva Dantas et al. 2010; Malagnac et al. 2007). Addition of the reducing agents DTT or ascorbic acid should balance highly oxidized intracellular conditions that might exist in $\Delta$ trr 1 but could not compensate its growth defect, in contrast to transcription factor Ltf1 (Schumacher et al. 2014). Accordingly, the growth retardation of $\Delta$ bctrr 1 seems to be caused by other factors.

In contrast to $\Delta$ bctrx 1 , the bctrrl knockout also showed enhanced ROS production. Moreover, sensitivity to the membraneactive substance SDS was shown clearly. A higher ROS pro- 
duction could lead to unstable membranes and to a restriction in growth, consequently making the fungus even more sensitive to stressors like peroxides.

As the phenotype of $\Delta$ bctrrl was more severe compared with $\Delta$ bctrx 1, the question arose why the deletion of the reductase has a stronger phenotype than the deletion of the thioredoxin. A redundant function of other thioredoxins compensating the absence of BcTrx1 was assumed. In organisms such as $S$. cerevisiae and $P$. anserina, especially in higher plants, elevated numbers of thioredoxins are present in various cellular compartments (Malagnac et al. 2007; Meyer et al. 2009). In B. cinerea, one thioredoxin reductase (BcTrr1) and several thioredoxin-like proteins could be identified. However, only one of these candidates was identified as a thioredoxin (BcTrx1). Based on sequence analysis, another putative second thioredoxin (BcTrx2) was found that contains the characteristic hallmark sequence CXXC. Due to structural differences, a participation of BcTrx 2 in the redox processes of the thioredoxin system is questionable. BcTrx2 was proved to be located in the ER, while BcTrx1 and BcTrr1 were shown microscopically to be cytosolic. Organisms have evolved quite specialized thioredoxins, located either in the cytosol, mitochondria, plastids, or even in the nucleus or at the plasma membrane (Arner and Holmgren 2000; Hirota et al. 1999; Martin and Dean 1991; Pedrajas et al. 1999; Serrato et al. 2013). In mammalian cells, a transmembrane thioredoxin-like protein (TMX4) was identified in the ER that has reductase activity in vitro and probably enables protein folding in cooperation with known proteins of the folding complex (Sugiura et al. 2010). As is predicted for BcTrx2, TMX4 has an ER-targeting signal, a transmembrane domain, and a thioredoxin domain facing the ER lumen (data not shown). Therefore, this role may also be ascribed to Botrytis Trx2. Bellisola and associates (2004) demonstrated an interaction of mammalian TrxR with an ER enzyme involved in oxidative protein folding, the disulfide isomerase (PDI). Although they argued that it is doubtful for a cytosolic protein to reduce an ER protein, they could show that TrxR can lead to a reduction or activation of ricin and immunotoxins in vivo via the activation of disulfide reductase activity of PDI (Bellisola et al. 2004).

The identified BcTrx2 was analyzed for its effects on development and growth on different media, $\mathrm{H}_{2} \mathrm{O}_{2}$ production, and virulence. However, no altered phenotype could be attributed to the deletion mutant. BcTrx2, therefore, seems to have no redundant functions, as it cannot compensate for the absence of bctrxl in the knockout mutant. At least, it does not seem to directly influence the redox or pathogenicity process. The colony diameters of a bctrx 1/2 double deletion on CM were shown to be reduced as compared with bctrxl and bctrx 2 single knockouts (Fig. 2A), but enhanced ROS production could not be detected. The phenotype of the bctrx $1 / 2$ double deletion conforms to the phenotype of $\Delta$ bctrr1 only in some aspects. BcTrr1 obviously does have additional, even more essential, enzymatic properties than BcTrx1 and BcTrx2. In mammals, it is postulated that thioredoxin reductases have multifaceted roles beyond the reduction of thioredoxin. Many splice variants have been described, the variants taking part in diverse signaling processes and numerous redox-related aspects of diseases (Arner 2009). The divergent phenotypes might also be explained by a gain of function of $\Delta$ bctrr 1 , as could be shown for $\Delta$ trrB in Escherichia coli (Stewart et al. 1998; Toledano et al. 2013). Stewart and associates (1998) revealed that, in the absence of $\operatorname{trr} B$, the oxidized thioredoxins $\operatorname{Trx} A$ and $\operatorname{TrxB}$ directly catalyze disulfide bond formation, an effect that is the reversal to their usual physiological function. In the $\Delta$ bctrr 1 mutant, $g p x$, grx, and glrl are highly expressed, indicating at least some overlapping and backup functions of the GSH and thioredoxin systems, though our data do not support this theory. Other OSR genes, such as cat 2 and prx, are constitutively expressed in $\Delta$ bctrrl. It is striking that the OSR genes deregulated in $\Delta b c t r r l$ are all target genes of Bap1 (Temme and Tudzynski 2009). According to the ROS assays, $\Delta$ bctrr1 produces large amounts of ROS, which could lead to accumulation of Bap1 in the nucleus, as reported for Yap1 in sctrx 1/2 double deletion mutants of $S$. cervisiae (Izawa et al. 1999).

OSR is not exclusively regulated by Bap1. It was shown that the transcription factor shares some target genes with the stressactivated mitogen-activated protein kinase (MAPK) BcSak1. $\mathrm{BcSak} 1$ is involved in vegetative growth and pathogenicity. Additionally, it is activated under conditions of oxidative and osmotic stress (Segmuller et al. 2007; Heller et al 2013). Therefore, it is likely that ROS play an activating role for the MAPK cascade. In mammals, the apoptosis signal-regulating kinase 1 (Ask1), a MAPK kinase kinase, was identified to be an upstream regulator of the JNK and p38 stress-activated protein kinases and plays a crucial role in cellular response to different stress conditions (Hayakawa et al. 2006; Ichijo et al. 1997). Thioredoxin was shown to inhibit the kinase activity of Ask1. Its reduced form directly binds to the $\mathrm{N}$-terminal region of Ask1 and thereby acts as negative regulator. In the presence of ROS, the reduced thioredoxin is converted to the oxidized form and dissociates from Ask1 (Saitoh et al. 1998). The release of Ask1 results in a conformational change and immediate autophosphorylation of the kinase domain (Tobiume et al. 2002). However, in B. cinerea, the phosphorylation level of BcSak 1 is not affected by loss of components of the thioredoxin system, therefore ruling out that this system has any impact on this MAPK cascade (data not shown).

To gain more information about the possible coherence between GSH and the thioredoxin system, roGFP2 was used as a sensor to measure the redox potential of the intracellular GSH pool. Lack of the key enzyme (BcGlr1) for GSH reduction resulted in an accumulation of GSSG after stress induction that could not be reduced physiologically. As BcGlr1 is an enzyme with high activity that is thought to be specific for GSSG, it was not surprising that the GSH pool remained highly oxidized in the deletion mutant. This approach confirms that BcGlr1 is the only reductase to recycle GSSG. The deletion of bctrx 1 had no influence on the system, either indicating that this enzyme is redundant or that thioredoxins generally do not affect components of the GSH system or the general redox milieu. Heller and associates (2012) tested the influence of the bapl deletion on the GSH level. As Bap1 was shown to control gene expression of components of the GSH pathway (Temme and Tudzynski 2009), the biosensor roGFP2 was introduced in the mutant to prove whether the absence of the oxidative stress responsive factor has an influence on GSH homeostasis. Only after repeated induction with $\mathrm{H}_{2} \mathrm{O}_{2}$ was the mutant no longer able to fully reduce GSH to the base level. This indicated a basal expression of the glr genes sufficient for a single reduction process but not for repeated recycling. Even repeated oxidative challenging of $\Delta$ bctrx $1:$ grx-rogfp2 did not affect the potential to reduce GSSG, confirming the assumption that BcTrx 1 is dispensable for GSH redox homeostasis.

As members of Trx fold-containing proteins, glutaredoxins share major protein functions with thioredoxins, such as the cleavage of disulfide bridges of substrates. However, in contrast to thioredoxins, glutaredoxins are reduced nonenzymatically by GSH (Holmgren 1989; Meyer et al. 2009). S. cerevisiae grx knockout mutants were described as sensitive to oxidative stress, underscoring the role of the GSH system in redox control (Luikenhuis et al. 1998). Our results show that BcGrx is not able to fully complement the loss of BcTrx 1 in B. cinerea, but still, it is possible that the systems at least share some over- 
lapping functions and targets. At this point, it would be interesting to see how a double deletion mutant, $\Delta$ bcgrx $1 /$ bctrx 1 , behaves. Glutaredoxin contributes to ROS scavenging and was even described to function as peroxidase itself (Collinson and Grant 2003).

During revision of this manuscript, a functional analysis of the thioredoxin and GSH systems in the model plant pathogen M. oryzae appeared (Fernandez and Wilson 2014). It showed that, in this hemibiotrophic pathogen, both redox systems have an essential role in virulence. Mutants lacking functional thioredoxin reductase were impaired in sporulation, appressoria development, and penetration (in contrast to B. cinerea), cellto-cell movement, and full symptom development. However, while we could find only a minor effect of the GSH reductase on virulence in $B$. cinerea, in $M$. oryzae also the GSH system has high impact on virulence. In contrast to thioredoxin, however, glutaredoxin is not essential for appressoria development and it seems to be necessary for suppression of ROS accumulation by the host. These differences probably reflect the different pathogenic strategies: $B$. cinerea is a necrotrophic fungus lacking the initial biotrophic phase characteristic for $M$. oryzae. A highly interesting aspect of this paper, however, links both systems: the authors show that glucose sensing is a prerequisite for activation of these antioxidant systems. In $B$. cinerea the standard inoculation procedure with conidial suspensions requires the presence of glucose. It will be interesting to test if also in this necrotrophic system such a link between primary metabolism and virulence via the activation of redox systems is operative.

In summary we could show that the thioredoxin system of Botrytis cinerea plays an essential role in developmental and virulence associated processes, whereas GSH reductases have only a minor function in these processes. Data presented in this work show the importance of intracellular redox balance and open new perspectives for the understanding of redox processes in host-pathogen interactions.

\section{MATERIALS AND METHODS}

\section{Fungal strains.}

The B05.10 strain of Botrytis cinerea Pers.:Fr. [Botryotinia fuckeliana (de Bary) Whetzel] is an isolate from a wild type of Vitis vinifera (Büttner et al. 1994). It serves as wild-type control for all experiments; all other strains used in this study are listed in Supplementary Table S1.

\section{Media and culture conditions.}

The cultivation of $S$. cerevisiae was carried out on complex media $(0.5 \%(\mathrm{wt} / \mathrm{vol})$ yeast extract, $2 \%(\mathrm{wt} / \mathrm{vol})$ glucose, $2 \%$ (wt/vol) peptone, $\mathrm{pH} 5.8)$ or selective drop-out (SD) media (20 $\mathrm{g}$ of glucose per liter, $6.7 \mathrm{~g}$ of Difco Yeast nitrogen base without amino acids per liter [BD, Franklin Lakes, NJ, U.S.A.], $0.77 \mathrm{~g}$ DO-supplement per liter [Clontech, Mountain View, CA, U.S.A.], $\mathrm{pH}$ 5.8). The incubation took place at $30^{\circ} \mathrm{C}$ and, for liquid media, additionally, $200 \mathrm{rpm}$. Strains containing plasmids were incubated on plates lacking the respective amino acids for selection. E. coli Top10F' (Invitrogen, San Diego, CA, U.S.A.) strains were cultivated in lysogeny broth media (in grams per liter, 10 of peptone, 5 of yeast extract, 10 of $\mathrm{NaCl}$ ) (Miller 1972 ) at $37^{\circ} \mathrm{C}$ and, for liquid media, additionally, at $180 \mathrm{rpm}$. The cultivation of $B$. cinerea was done on synthetic CM according to Pontecorvo and associates (1953) or on potato dextrose agar (PDA) (Sigma-Aldrich Chemie, Steinheim, Germany) supplemented with $100 \mathrm{~g}$ of homogenized leaves of French beans (PDAB) (Phaseolus vulgaris) per liter. Sporulation was triggered by light exposure and incubation for 7 days at 18 to $20^{\circ} \mathrm{C}$. For certain experiments, the strains were also cultivated in $\mathrm{CD}$ (in grams per liter: 20 of sucrose, 3 of $\mathrm{NaNO}_{3}, 1$ of $\mathrm{K}_{2} \mathrm{HPO}_{4}, 0.5$ of $\mathrm{KCl}, 0.01$ of $\mathrm{FeSO}_{4} \times 7 \mathrm{H}_{2} \mathrm{O}, 0.5$ of $\mathrm{MgSO}_{4} \times 7 \mathrm{H}_{2} \mathrm{O}$, pH 5.2) or in Gamborg's medium (Duchefa Biochemie BV, Haarlem, The Netherlands) supplemented with $2 \%$ glucose. For isolation of genomic DNA, the fungus was grown on solid CM medium overlaid with cellophane for about 2 to 3 days. Stress-sensitivity assays were performed by inoculating 3-day-old mycelial plugs derived from CM plates on CM plates supplemented with different stressors. For isolation of RNA or proteins, $B$. cinerea was grown in axenic culture under diverse stress conditions, as indicated. The strains were grown for 3 days on CM medium covered with cellophane. The mycelium was homogenized and was used for the inoculation of $1.5 \%$ malt medium. After $14 \mathrm{~h}$ at $18^{\circ} \mathrm{C}$ and 150 rpm, the mycelium was harvested via Nytex filtration and was shifted into modified minimal CD medium (in grams per liter: 20 of glucose, 1 of $\mathrm{NaNO}_{3}, 1$ of $\mathrm{K}_{2} \mathrm{HPO}_{4}, 0.5$ of $\mathrm{KCl}, 0.5$ of $\mathrm{MgSO}_{4} \times 7 \mathrm{H}_{2} \mathrm{O}, \mathrm{pH}$ 5.2). Following an additional incubation for $24 \mathrm{~h}, \mathrm{CD}$ medium including stress-inducing supplements $\left(\mathrm{H}_{2} \mathrm{O}_{2}\right.$ or $\left.\mathrm{NaCl}\right)$ was inoculated with $3 \mathrm{~g}$ of mycelium and was incubated at $18^{\circ} \mathrm{C}$ and $150 \mathrm{rpm}$ for another $30 \mathrm{~min}$.

\section{Transformation.}

For the deletion of bctrr $1, b c t r x 2$, and $b c g l r 2$, the $5^{\prime}$ gene flank $\left(1,000,1,200\right.$, and $625 \mathrm{bp}$, respectively) and the $3^{\prime}$ gene flank (966, 850, and $913 \mathrm{bp}$ ) were amplified from the genomic DNA of B. cinerea B05.10 using primers 3/4 and 5/6, 27/28 and 29/30, and 43/44 and 45/46 (Supplementary Table S2). As selection marker, the $h p h$ cassette from the vector pCSN44 was used (primer 11/12). The cassette contains the constitutive $\operatorname{trp} C$ promoter of $A$. nidulans and the $h p h$ gene. For the bctrxl and bcglrl deletion construct, flanking regions of 936 to 1,074 bp and 814 to 945 bp were amplified, using primers $15 / 16$ and $17 / 18$ and $35 / 36$ and $37 / 38$. The nourseothricin resistance cassette from the vector pNR1 was used as selection marker and was amplified with primer pair 23/24. The nat1 gene is under control of the oliC promoter of $A$. nidulans. The deletion vectors were constructed using the yeast recombinational cloning system (Colot et al. 2006) via the uracil-auxotrophic strain $S$. cerevisiae FY 834 (Winston et al. 1995), which was co-transformed with the amplified fragments and the EcoRI/XhoIdigested plasmid pRS426. The selection on SD media lacking uracil enables only cells that contain the recombined circular vector to grow, given that the uracil auxotrophy of the yeast strain is complemented. After cultivation of uracil prototrophic yeast transformants, the DNA was isolated using the GeneJETPlasmid miniprep kit (Fermentas, Vilnius, Lithuania), including an initial step of disrupting yeast cells by the addition of glass beads. Subsequently, the respective deletion fragments were amplified by PCR, using the primer combination 3/6 (bctrrl), 15/18 (bctrxl), 27/30 (bctrx2), 35/38 (bcglrl), and 43/46 (bcglr2) and was used for the transformation of $B$. cinerea B05.10 protoplasts.

Homologous integration (HI) of the resistance cassettes at the gene locus of interest was proved by diagnostic PCR. For 3' and $5^{\prime}$, HI primers were used that bind outside of the gene flanks and within the resistance cassette. Untransformed DNA was detected by using primers located in the gene of interest. Southern blot analysis was performed to exclude ectopic integrations.

In order to prove that the phenotype of the mutants is due to the deletion of a single gene, a complementation approach was done to rescue the B05.10 wild-type phenotype. Via yeast recombinational cloning (Colot et al. 2006), the complementation fragments were constructed, containing the entire ORF of bctrx 1, bctrx 2 , and bctrr 1 , respectively. Due to the high affinity of $B$. cinerea to homologous integration, targeted gene disruption of an expandable gene was used to complement the de- 
letion strains. A suitable vector system for $B$. cinerea has been designed using the nitrite reductase locus (BcNiiA; Johnstone et al. 1990; Schumacher 2012). As long as the fungus grows on media containing a nitrogen source other than nitrate, it will not be affected by deletion of this gene. The vector $\mathrm{pNAH} / \mathrm{N}$ OGG contains the vector backbone of pRS426, the $5^{\prime}$, and the $3^{\prime}$ flank of bcniiA, a resistance cassette with $\operatorname{trp} C$ promoter of A. nidulans, and an expression cassette (PoliC-ogfp-Tgluc) (Schumacher 2012). For $\Delta$ bctrr 1 and $\Delta$ bctrx 2 complementation, the vector pNAN-OGG containing the nourseothricin-resistance gene natl was used and, for $\Delta b c t r x l$ complementation, the vector pNAH-OGG containing the hygromycin-resistance gene $h p h$. The vectors were digested with NotI and SpeI, in order to cut out the ogfp expression cassette. The BcTrr1, BcTrx1, and BcTrx 2 ORF, including $1 \mathrm{~kb}$ of the particular promoter and $0.5 \mathrm{~kb}$ of the particular terminator sequences, were amplified by PCR, based on genomic DNA with primers 49/50, 51/52, and 53/54, resulting in $2.9,2.3 \mathrm{~kb}$, and $2,4 \mathrm{~kb}$ PCR fragments, respectively. Overlapping ends to the $5^{\prime}$ bcniiA flank and the $\operatorname{trp} C$ promoter were included in the primer sequences to ensure a homologous recombination of the inserts with the vector.

For the generation of the bctrx1-gfp, bctrr1-gfp, bctrx2-gfp, bcglr1-gfp, and bcglr2-gfp fusion and complementation constructs, the respective genes were amplified using primers $55 / 56$, $57 / 58,59 / 60,61 / 62$, and 63/64, respectively. These primers contain sequences homologous to the glucanase terminator and the $g f p$ of the vector pNDH-OGG/pNDN-OGG (Schumacher 2012). The PCR products were co-transformed into the uracilauxotrophic strain $S$. cerevisiae FY834, with pNDH-OGG and pNDN-OGG linearized with NotI or pNDN-OGG linearized with NcoI, as described above. The plasmid DNA from uracilprototrophic yeast colonies was isolated using the SpeedPrep yeast plasmid isolation kit (DualsystemsBiotech, Schlieren, Switzerland) and was transformed into E. coli. Following plasmid isolation, the expression cassette was sequenced and excised using the enzymes ApaI and SacII and was transformed into B. cinerea $\mathrm{B} 05.10$.

Furthermore, the vector pNAH::grx-rogfp2 (Heller et al. 2012) was digested with HindIII and SacII and was used for the transformation of $\Delta$ bctrx 1 and $\Delta$ bcglr 1 . For $\Delta$ bctrr 1 , a new vector was constructed, containing the nourseothricin-resistance cassette (pNAN::grx-rogfp2).

For the transformation of B. cinerea, $1.5 \mathrm{~g}$ of mycelium was weighted and mixed with a 10-ml enzyme mixture of glucanex (Novozymes, Bagsværd, Denmark), lysing enzyme (SigmaAldrich, St. Louis), and Yatalase (TakaraBio Inc., Shiga, Japan) and was incubated for $1 \mathrm{~h}$ at $28^{\circ} \mathrm{C}$ at $95 \mathrm{rpm}$. Protoplasts were transformed according to Schulze-Gronover and associates (2001), using $20 \mu \mathrm{g}$ of the linearized vector or $300 \mu \mathrm{l}$ of PCR products. Resistant colonies were transferred to agar plates containing GB5 agar and supplemented with $70 \mu \mathrm{g}$ of hygromycin $\mathrm{B}$ per milliliter (Invitrogen) or $70 \mu \mathrm{g}$ of nourseothricin per milliliter (Werner-Bioagents, Jena, Germany). Conidiospores of heterokaryotic transformants were harvested, diluted with sterile $\mathrm{H}_{2} \mathrm{O}$ and 50 to 100 spores plated on selection medium. After $24 \mathrm{~h}$, germinated single spores were picked and transferred to new selection medium. The success of isolation was controlled by extraction of genomic DNA, followed by a diagnostic PCR and Southern blots.

\section{Standard molecular methods.}

For isolation of genomic DNA of $B$. cinerea, a modified protocol of Cenis (1992) was used.

Southern blot analysis was performed according to Sambrook and associates (1989). The radioactive labeling of probes used for hybridizations was carried out with the Random primed la- beling kit, purchased from Boehringer (Ingelheim, Germany), according to the manufacturer's protocol. This kit is based on a method described by Feinberg and Vogelstein (1983), in which $\alpha-\left[{ }^{32} \mathrm{P}\right] \mathrm{dCTP}$ is incorporated into newly synthesized DNA strands. The reaction took place for 1 to $2 \mathrm{~h}$ at $37^{\circ} \mathrm{C}$. Free nucleotides were separated via gel filtration on a Sephadex G-50 column, as described by Ausubel and associates (1987). Subsequently the probes were denaturized for $5 \mathrm{~min}$ at $95^{\circ} \mathrm{C}$ and were cooled on ice prior to hybridization performed according to Sambrook and associates (1989). In order to block free binding sites, membranes were prehybridized for 1 to $2 \mathrm{~h}$ at $65^{\circ} \mathrm{C}$ in $6 \times \mathrm{SSPE}\left(1 \times \mathrm{SSPE}\right.$ is $0.18 \mathrm{M} \mathrm{NaCl}, 10 \mathrm{mM} \mathrm{NaPO}_{4}$, and $1 \mathrm{mM}$ EDTA, pH 7.7), $5 \times$ Denhardt's solution, $0.1 \%$ SDS, and $50 \mathrm{mM}$ phosphate buffer, $\mathrm{pH}$ 6.6, containing salmon sperm DNA. The actual hybridization was done overnight at $65^{\circ} \mathrm{C}$. Unspecific bound probes were removed by washing for 5 to $10 \mathrm{~min}$ at $65^{\circ} \mathrm{C}$ with $2 \times \mathrm{SSC}(1 \times \mathrm{SSC}$ is $0.15 \mathrm{M} \mathrm{NaCl}$ plus $0.015 \mathrm{M}$ sodium citrate), $0.1 \% \mathrm{SDS}$ and for $10 \mathrm{~min}$ in $1 \times$ SSC, $0.1 \%$ SDS.

RNA isolation took place through phenol-chloroform extraction using the Trizol reagent (Invitrogen) according to the manufacturer's instructions. For Northern analysis, 15- $\mu \mathrm{g}$ RNA samples were separated in a $1 \%$ agarose gel containing formaldehyde and were then transferred by downward blotting (Ausubel et al. 1987) to a nylon transfer membrane (Nytran SPC Whatman, Schleicher and Schuell, Dassel, Germany) according to Sambrook and associates (1989). Blot hybridizations were done in $0.6 \mathrm{M} \mathrm{NaCl}, 0.16 \mathrm{M} \mathrm{Na}_{2} \mathrm{HPO}_{4}, 0.06 \mathrm{M}$ EDTA, 1\% N-lauroylsarcosine (Sigma-Aldrich), 10\% dextran sulfate at $\mathrm{pH}$ 6.2, as described for Southern blots.

\section{Conidial germination.}

To analyze nutrient-dependent spore germination, conidiospores (Doehlemann et al. 2006) were harvested from CM or PDA + bean agar plates and were filtrated over Nytex. The spore titer was adjusted to $1 \times 10^{5}$ spores per milliliter. Droplets $(10 \mu \mathrm{l})$ of spore solution were dropped on a cover slip and were incubated in the dark for $24 \mathrm{~h}$. In order to determine the germination rate on a hydrophobic surface, the spores were resuspended in $1 \mathrm{ml}$ of sterile $\mathrm{H}_{2} \mathrm{O}$ and were washed several times to remove all nutrients; $10-\mu l$ drops were placed on polypropylene foil fixed on cover slips. The germination progress was monitored by light microscopy.

\section{ROS staining.}

For the analysis of exogenous $\mathrm{H}_{2} \mathrm{O}_{2}$, strains were grown on $\mathrm{CM}$ overlaid with cellophane for 3 days. Mycelium $(25 \mathrm{mg})$ was weighed and placed in $1 \mathrm{ml}$ of fresh DAB solution $(0.5$ $\mathrm{mg}$ of DAB per milliliter in $100 \mathrm{mM}$ citric acid, $\mathrm{pH}$ 3.7). After incubation for $1.5 \mathrm{~h}$ in the dark at room temperature, the mycelium was removed and the coloration was recorded photographically. As negative control, either DAB solution or DAB supplemented with $1 \mu \mathrm{l}$ of $\mathrm{H}_{2} \mathrm{O}_{2}(30 \%)$ was used. For the positive control, $2 \mu \mathrm{l}$ of horseradish peroxidase and $1 \mu \mathrm{l}$ of $\mathrm{H}_{2} \mathrm{O}_{2}$ in different dilutions were added to the DAB.

The analysis of superoxide production was done according to Egan and associates (2007). Droplets $(10 \mu \mathrm{l})$ at a concentration of $10^{5}$ spores per milliliter were placed on cover slips and were germinated in a moist chamber. For staining, a $0.05 \%$ (wt/vol) NBT aqueous solution was added to the germinated conidia. After incubation for $1 \mathrm{~h}$, the formazan staining was monitored by light microscopy. As a control, $50 \mu \mathrm{M}$ DPI was added prior to the addition of NBT.

\section{Pathogenicity and penetration assay.}

The pathogenicity assay for the characterization of different $B$. cinerea strains was performed as described by Klimpel and 
associates (2002). Conidiospores were harvested from 10-dayold PDAB plates, and the spore titer was adjusted to $2 \times 10^{5}$ spores per milliliter. For the assay, 7.5- $\mu 1$ drops were placed on primary foliage bean leaves of Phaseolus vulgaris L. genotype N90598 (originating from J. D. Kelly, Michigan State University, East Lansing, MI, U.S.A.). The incubation of plants took place under humid conditions at $20^{\circ} \mathrm{C}$.

In order to see whether $B$. cinerea spores are able to penetrate onion epidermal cells, conidiospores were harvested from $\mathrm{CM}$ or PDA+ bean agar plates. They were then washed with sterile double-distilled water, and the spore titer was adjusted to $1 \times 10^{5}$ spores per milliliter. The onion epidermis was also washed with sterile double-distilled water and were placed hydrophobic side up on a glass slide. The slides were put in a humid chamber and were incubated for $1 \mathrm{~h}$ at $70^{\circ} \mathrm{C}$. For inoculation, 10-ml drops or agar plugs were placed on the epidermis. The incubation of slides took place for $24 \mathrm{~h}$ in a humid chamber. For monitoring hyphae on top of the epidermis, the stain lactophenol blue was used and the penetration ability was evaluated by light microscopy after $5 \mathrm{~min}$.

\section{Epifluorescence microscopy.}

Light and fluorescence microscopic analyses occurred by using the Axio Imager 2 from Zeiss (Jena, Germany), using $40 \times$ or $63 \times$ objectives or the inverse microscope Observer Z.1 (Zeiss, Jena, Germany) using a $20 \times$ objective lens.

Hoechst33342 staining was examined using the filter set 49 DAPI shift free (excitation G 365, beam splitter FT 395, emission BP 445/50), GFP fluorescence was detected with filter set 38 (excitation BP 470/40, beam splitter FT 495, emission BP 525/50). Images were captured with a Zeiss AxioCam MRm camera and were analyzed using the Axiovision Rel 4.8 software package.

\section{SEM.}

For the analysis of fungal germination and penetration on plant material, conidiospores were harvested, the spore titer was adjusted to $2 \times 10^{6}$ spores per milliliter and was sprayed on bean leaves. After 16 to $18 \mathrm{~h}$ at $20^{\circ} \mathrm{C}$, squares $\left(0.5 \mathrm{~cm}^{2}\right)$ were excised and fixed with $3 \%$ glutaraldehyde. For dehydration of the samples, an ethanol concentration series was applied as described by Giesbert and associates (1998). Critical point drying took place following the standard protocol (Emitech K850 Critical Point dryer; Emitech, Ashford, England), in which carbon dioxide was used for supercritical drying. Afterwards, the dried samples were mounted on aluminum pads and were coated with gold (Emitech vacuum sputter device K5506). For microscopic monitoring, a Hitachi S-3000N scanning electron microscope was used at $15 \mathrm{kV}$.

\section{Measurement of the intracellular GSH pool.}

For fluorometer-based measurements, conidia from sporulated PDA + bean plates were used to inoculate $100 \mathrm{ml}$ of malt medium. After $12 \mathrm{~h}$ at $18^{\circ} \mathrm{C}$ and $150 \mathrm{rpm}, 2-\mathrm{ml}$ samples were taken and washed twice in GB5 with $1 \mathrm{mM}(\mathrm{NH})_{4} \mathrm{HPO}_{4}$. Then, $200 \mu \mathrm{l}$ of germinated spores were transferred to a 96-well plate (Microplate pureGrade 96-well PS, BRAND GMBH + CO KG, Wertheim am Main, Germany) and fluorescence was measured with $3 \times 3$ reads per well, using a Tecan Safire fluorometer (Tecan Group Ltd., Männedorf, Switzerland). For the oxidized state, an excitation wavelength of $395 \mathrm{~nm}$ was used and, for the reduced state, a wavelength of $488 \mathrm{~nm}$; emission was detected at $510 \mathrm{~nm}$. The relative fluorescence units were used to calculate the $\mathrm{Em}_{395} / \mathrm{Em}_{488}$ ratio.

For the CLSM, glass slides with $10-\mu$ l droplets of conidia suspension $\left(1 \times 10^{5}\right.$ spores per milliliter of GB5) were prepared and were left to germinate overnight. The microscopic analysis was done using an inverted microscope (Leica DMIRE2), according to Heller and associates (2012). To calculate the image data radiometric analyses using ImageJ (v. 1.44f) was done.

\section{ACKNOWLEDGMENTS}

We thank B. Williamson for critically reading the manuscript, U. Siegmund and A. Antoni for technical support and discussions, and the Deutsche Forschungsgemeinschaft (DFG) (Tu 50/17; A.Viefhues and N. Temme were funded by the DFG graduate school 1409) for funding.

\section{LITERATURE CITED}

Aguirre, J., Rios-Momberg, M., Hewitt, D., and Hansberg, W. 2005. Reactive oxygen species and development in microbial eukaryotes. Trends Microbiol. 13:111-118.

Arner, E. S. 2009. Focus on mammalian thioredoxin reductases-Important selenoproteins with versatile functions. Biochim. Biophys. Acta. 1790:495-526.

Arner, E. S., and Holmgren, A. 2000. Physiological functions of thioredoxin and thioredoxin reductase. Eur J Biochem. 267:6102-6109.

Ausubel, F.M., Brent, R., Kingston, R.E., Moore, D.D., Seidman, J.G., Smith, J.A., et al. 1987. Current Protocols in Molecular Biology. John Wiley and Sons, New York.

Beckmann, K. B., and Ames, B. N. 1998. The free radical theory of aging matures. Physiol. Rev. 78:547-581.

Bellisola, G., Fracasso, G., Ippoliti, R., Menestrina, G., Rosen, A., Solda, S., Udali S., Tomazzolli R., Tridente G., and Colombatti M. 2004. Reductive activation of ricin and ricin A-chain immunotoxins by protein disulfide isomerase and thioredoxin reductase. Biochem. Pharmacol. 67:1721-1731.

Belozerskaia, T. A., and Gessler, N. N. 2007. Reactive oxygen species and the strategy of the antioxidant defense in fungi: A review. Prikl. Biokhim. Mikrobiol. 43:565-575.

Bjur, E., Eriksson Ygberg, S., Aslund, F., and Rhen, M. 2006. Thioredoxin 1 Promotes intracellular replication and virulence of Salmonella enterica serovar Typhimurium. Infect Immun. 74:5140-5151.

Brandes, H. K., Larimer, F. W., Geck, M. K., Stringer, C. D., Schurmann, P., and Hartman, F. C. 1993. Direct identification of the primary nucleophile of thioredoxin f. J. Biol. Chem. 268:18411-18414.

Camier, S., Ma, E., Leroy, C., Pruvost, A., Toledano, M., and MarsolierKergoat, M. C. 2007. Visualization of ribonucleotide reductase catalytic oxidation establishes thioredoxins as its major reductants in yeast. Free Radical Bio. Med. 42:1008-1016.

Cenis, J. L. 1992. Rapid extraction of fungal DNA for PCR amplification. Nucleic Acids Res. 20:2380.

Chen, K., Craige, S. E., and Keaney, J. F., Jr. 2009. Downstream targets and intracellular compartmentalization in Nox signaling. Antioxid Redox Signal. 11:2467-2480.

Collinson, E. J., and Grant, C. M. 2003. Role of yeast glutaredoxins as glutathione S-transferases. J. Biol. Chem. 278:22492-22497.

Colot, H. V., Park, G., Turner, G. E., Ringelberg, C., Crew, C. M., Litvinkova, L., Weiss, R. L., Borkovichm K. A., and Dunlap, J, C. 2006. A high-throughput gene knockout procedure for Neurospora reveals functions for multiple transcription factors. Proc. Natl. Acad. Sci. U.S.A. 103:10352-10357.

da Silva Dantas, A., Patterson, M. J., Smith, D. A., Maccallum, D. M., Erwig, L. P., Morgan, B. A., and Quinn, J. 2010. Thioredoxin regulates multiple hydrogen peroxide-induced signaling pathways in Candida albicans. Mol. Cell Biol. 30:4550-4563.

Doehlemann, G., Berndt, P., and Hahn, M. 2006. Different signalling pathways involving a Galpha protein, cAMP and a MAP kinase control germination of Botrytis cinerea conidia. Mol. Microbiol. 59:821835 .

Doehlemann, G., Molitor, F., and Hahn, M. 2005. Molecular and functional characterization of a fructose specific transporter from the gray mold fungus Botrytis cinerea. Fungal Genet. Biol. 42:601-610.

Egan, M. J., Wang, Z. Y., Jones, M. A., Smirnoff, N., and Talbot, N. J. 2007. Generation of reactive oxygen species by fungal NADPH oxidases is required for rice blast disease. Proc. Natl. Acad. Sci. U.S.A. 104:1177211777.

Elad, Y., and Evenses, K. 1995. Physiological aspects of resistance to Botrytis cinerea. Phytopathology 85:637-643.

Feinberg, A. P., and Vogelstein, B. 1983. A technique for radiolabeling DNA restriction endonuclease fragments to high specific activity. Anal. Biochem. 132:6-13.

Fernandez, J., and Wilson, R. 2014. Characterizing Roles for the glutathione reductase, thioredoxin reductase and thioredoxin peroxidase-encod- 
ing genes of Magnaporthe oryzae during rice blast disease. PLoS One. 9(1):e87300. Published online.

Foyer, C. H., and Noctor, G. 2005. Redox homeostasis and antioxidants signaling: A metabolic interface between stress perception and physiological responses. Plant Cell 17:1866-1875

Garrido, E. O., and Grant, C. M. 2002. Role of thioredoxins in the response of Saccharomyces cerevisiae to oxidative stress induced by hydroperoxides. Mol. Microbiol. 43:993-1003.

Giesbert, S., Lepping, H. B., Tenberge, K. B., and Tudzynski, P. 1998. The Xylanolytic system of Claviceps purpurea: Cytological evidence for secretion of xylanases in infected rye tissue and molecular characterization of two xylanase genes. Phytopathology 88:1020-1030.

Gonzalez Porque, P., Baldesten, A., and Reichard, P. 1970. The involvement of the thioredoxin system in the reduction of methionine sulfoxide and sulfate. J. Biol. Chem. 245:2371-2374.

Govrin, E. M., and Levine, A. 2000. The hypersensitive response facilitates plant infection by the necrotrophic pathogen Botrytis cinerea. Curr Biol. 10:751-757.

Groves, J. W., and Loveland, C. A. 1953. The connection between Botryotinia fuckeliana and Botrytiscinerea. Mycologica. 45:415-425.

Hayakawa, T., Matsuzawa, A., Noguchi, T., Takeda, K., and Ichijo, H. 2006. The ASK1-MAP kinase pathways in immune and stress responses. Microbes Infect. 8:1098-1107.

Heller, J., and Tudzynski, P. 2011. Reactive Oxygen species in phytopathogenic fungi: Signaling, development and disease. Ann. Rev. Phytopathol. 49:16.1-16.22.

Heller, J., Meyer, A. J., and Tudzynski, P. 2012. Redox-sensitive GFP2: Use of the genetically encoded biosensor of the redox status in the filamentous fungus Botrytis cinerea. Mol. Plant Pathol. 13:935-947.

Hirota, K., Murata, M., Sachi, Y., Nakamura, H., Takeuchi, J., Mori, K. and Yodoi, J. 1999. Distinct roles of thioredoxin in the cytoplasm and in the nucleus. A two-step mechanism of redox regulation of transcription factor NF-kappaB. J. Biol. Chem. 274:27891-27897.

Holmgren, A. 1985. Thioredoxin. Annu Rev Biochem. 54:237-271.

Holmgren, A. 1989. Thioredoxin and glutaredoxin systems. J Biol Chem. 264:13963-13966.

Holmgren, A., and Bjornstedt, M. 1995. Thioredoxin and thioredoxin reductase. Methods Enzymol. 252:199-208.

Ichijo, H., Nishida, E., Irie, K., ten Dijke, P., Saitoh, M., Moriguchi, T., Takagi, M., Matsumoto, K., Miyazono, K., and Gotoh, Y. 1997. Induction of apoptosis by ASK1, a mammalian MAPKKK that activates SAPK/JNK and p38 signaling pathways. Science. 275:90-94.

Izawa, S., Maeda, K., Sugiyama, K., Mano, J., Inoue, Y., and Kimura, A. 1999. Thioredoxin deficiency causes the constitutive activation of Yap1, an AP-1-like transcription factor in Saccharomyces cerevisiae. J. Biol. Chem. 274:28459-28465.

Johnstone, I. L., McCabe, P. C., Greaves, P., Gurr, S. J., Cole, G. E., Brow, M. A., Unkles, S. E., Clutterbuck, A. J., Kinghorn, J. R., and Innis, M. A. 1990. Isolation and characterisation of the crnA-niiA-niaD gene cluster for nitrate assimilation in Aspergillus nidulans. Genes 90:181192.

Jones, D.P. 2008. Radical-free biology of oxidative stress. Am. J. Physiol. Cell Physiol. 295:C849-68.

Kern, R., Malki, A., Holmgren, A., and Richarme, G. 2003. Chaperone properties of Escherichia coli thioredoxin and thioredoxin reductase. Biochem. J. 371:965-972.

Klimpel, A., Schulze-Gronover, C., Williamson, B., Stewart, J. A., and Tudzynski, B. 2002. The adenylate cyclase (BAC) in Botrytis cinerea is required for full pathogenicity. Mol. Plant Pathol. 3:439-450.

Leal, S. M., Jr, Vareechon, C., Cowden, S., Cobb, B. A., Latgé, J.-P., Momany, M., and Pearlman, E. 2012. Fungal antioxidant pathways promote survival against neutrophils during infection. J. Clin. Invest. 122:2482-2498

Luikenhuis, S., Perrone, G., Dawes, I. W., and Grant, C. M. 1998. The yeast Saccharomyces cerevisiae contains two glutaredoxin genes that are required for protection against reactive oxygen species. Mol. Biol. Cell 9:1081-1091.

Mahmood, D. F., Abderrazak, A., El Hadri, K., Simmet, T., and Rouis, M. 2013. The thioredoxin system as a therapeutic target in human health and disease. Antioxid. Redox. Sign. 19:1266-1303.

Malagnac, F., Klapholz, B., and Silar, P. 2007. PaTrx1 and PaTrx3, two cytosolic thioredoxins of the filamentous ascomycete Podospora anserina involved in sexual development and cell degeneration. Eukaryot. Cell 6:2323-2331.

Martin, H., and Dean, M. 1991. Identification of a thioredoxin-related protein associated with plasma membranes. Biochem. Biophys. Res. Commun. 175:123-128.

Martin, J. L. 1995. Thioredoxin-A fold for all reasons. Structure. 3:245250

Meyer, A. J. 2008. The integration of glutathione homeostasis and redox signaling. J. Plant Physiol. 165:1390-1403.

Meyer, A. J., Brach, T., Marty, L., Kreye, S., Rouhier, N., Jacquot, J. P., and Hell, R. 2007. Redox-sensitive GFP in Arabidopsis thaliana is a quantitative biosensor for the redox potential of the cellular glutathione redox buffer. Plant J. 52:973-986.

Meyer, Y., Buchanan, B. B., Vignols, F., and Reichheld, J. P. 2009. Thioredoxins and glutaredoxins: Unifying elements in redox biology. Annu. Rev. Genet. 43:335-367.

Muller, E. G. 1991. Thioredoxin deficiency in yeast prolongs S phase and shortens the G1 interval of the cell cycle. J Biol Chem. 266:91949202.

Mur, L. A. J. 2008. The hypersensitive response; the centenary is upon us but how much do we know? J Exp Bot. 59:501.

Mustacich, D., and Powis, G. 2000. Thioredoxin reductase. Biochem J. 346 Pt 1:1-8.

Pedrajas, J. R., Kosmidou, E., Miranda-Vizuete, A., Gustafsson, J. A. Wright, A. P., and Spyrou, G. 1999. Identification and functional characterization of a novel mitochondrial thioredoxin system in Saccharomyces cerevisiae. J. Biol. Chem. 274:6366-6373.

Powis, G., Mustacich, D., and Coon, A. 2000. The role of the redox protein thioredoxin in cell growth and cancer. Free Radical Bio. Med. 29:312-322.

Ravi, D., Muniyappa, H., and Das, K. C. 2005. Endogenous thioredoxin is required for redox cycling of anthracyclines and p53-dependent apoptosis in cancer cells. J. Biol. Chem. 280:40084-40096.

Rivas, S., and Thomas, C. M. 2005. Molecular interactions between tomato and the leaf mold pathogen Cladosporium fulvum. Annu. Rev. Phytopathol. 43:395-436.

Rolke, Y., Liu, S., Quidde, T., Williamson, B., Schouten, A., Weltring, K. M., Siewers, V., Tenberge, K. B., Tudzynski, B., and Tudzynski, P. 2004. Functional analysis of $\mathrm{H}(2) \mathrm{O}(2)$-generating systems in Botrytis cinerea: The major $\mathrm{Cu}-\mathrm{Zn}$-superoxide dismutase (BCSOD1) contributes to virulence on French bean, whereas a glucose oxidase (BCGOD1) is dispensable. Mol. Plant Pathol. 5:17-27.

Saitoh, M., Nishitoh, H., Fujii, M., Takeda, K., Tobiume, K., Sawada, Y., Kawabata, M., Miyazono, K., and Ichijo, H.1998. Mammalian thioredoxin is a direct inhibitor of apoptosis signal-regulating kinase (ASK) 1. EMBO (Eur. Mol. Biol. Organ.) J. 17:2596-2606.

Sambrook, J., Fritsch, E. F., and Maniatis, T. 1989. Molecular Cloning: A Laboratory Manual. 2nd edition. Cold Spring Harbor Laboratory Press. Cold Spring Harbor, NY, U.S.A.

Schouten, A., Tenberge, K. B., Vermeer, J., Stewart, J., Wagemakers, L., Williamson, B., and van Kan, J. A. 2002. Functional analysis of an extracellular catalase of Botrytis cinerea. Mol. Plant Pathol. 3:227-238.

Schulze-Gronover, C., Kasulke, D., Tudzynski, P., and Tudzynski, B. 2001. The role of $G$ protein alpha subunits in the infection process of the gray mold fungus Botrytis cinerea. Mol. Plant-Microbe Interact. 14:1293-1302.

Schumacher, J. 2012. Tools for Botrytis cinerea: New expression vectors make the gray mold fungus more accessible to cell biology approaches. Fungal Genet Biol. 49:483-497.

Schumacher, J., Simon, A., Cohrs, K. C., Viaud, M., and Tudzynski, P. 2014. The transcription factor BcLTF1 regulates virulence and light responses in the necrotrophic plant pathogen Botrytis cinerea. PLoS Genetics. doi: 10.1371/journal.pgen.1004040. Published online.

Segmuller, N., Ellendorf, U., Tudzynski, B., and Tudzynski, P. 2007. BcSAK1, a stress-activated mitogen-activated protein kinase, is involved in vegetative differentiation and pathogenicity in Botrytis cinerea. Eukaryot. Cell. 6:211-221.

Serrato, A. J., Fernandez-Trijueque, J., Barajas-Lopez, J. D., Chueca, A., and Sahrawy, M. 2013. Plastid thioredoxins: A "one-for-all" redox-signaling system in plants. Front Plant Sci. 4:463.

Shi, Y., Liu, L., Zhang, T., Shen, L., Zhang, J., Zhang, Y., Wang, X., Yang, S., Lu, F., Chen, X., and Ding, S. 2013. The involvement of Helicobac ter pylori thioredoxin-1 in gastric carcinogenesis. J. Med. Microbiol. 62:1226-1234.

Shlezinger, N., Minz, A., Gur, Y., Hatam, I., Dagdas, Y.F., Talbot, N. J., and Sharon, A. 2011. Anti-apoptotic machinery protects the necrotrophic fungus Botrytis cinerea from host-induced apoptotic-like cell death during plant infection. PLoS Pathog. 7:e1002185. Published online.

Stewart, E. J., Aslund, F., and Beckwith, J. 1998. Disulfide bond formation in the Escherichia coli cytoplasm: An in vivo role reversal for the thioredoxins. EMBO (Eur. Mol. Biol. Organ.) J. 17:5543-5550.

Sugiura, Y., Araki, K., Iemura, S., Natsume, T., Hoseki, J., and Nagata, K. 2010. Novel thioredoxin-related transmembrane protein TMX4 has reductase activity. J. Biol. Chem. 285:7135-7142.

Temme, N., and Tudzynski, P. 2009. Does Botrytis cinerea ignore $\mathrm{H}_{2} \mathrm{O}_{2}$ induced oxidative stress during infection? Characterization of Botrytis activator protein 1. Mol Plant Microbe Interact. 22:987-998.

Tenberge, K. B. 2007. Imaging of Infection. Pages 74-79 in: Botrytis: 
Biology, Pathology and Control. Y. Elad, B. Williamson, P. Tudzynski, and N. Delen, eds. Springer, Berlin

Tenberge, K. B., Beckedorf, M., Hoppe, B., Schouten, A., Solf, M., and von den Driesch, M. 2002. In situ localization of AOS in host-pathogen interactions. Microsc. Microanal. 8:250-251.

Tobiume, K., Saitoh, M., and Ichijo, H. 2002. Activation of apoptosis signal-regulating kinase 1 by the stress-induced activating phosphorylation of pre-formed oligomer. J. Cell Physiol. 191:95-104.

Toledano, M. B., Delaunay-Moisan, A., Outten, C. E., and Igbaria, A. 2013. Functions and cellular compartmentation of the thioredoxin and glutathione pathways in yeast. Antioxid. Redox. Sign. 18:1699-1711.

Torres, M. A., and Dangl, J. L. 2005. Functions of the respiratory burst oxidase in biotic interactions, abiotic stress and development. Curr Opin Plant Biol. 8:397-403.

Tudzynski, P., and Kokkelink, L. 2009. Botrytis cinerea: Molecular aspects of a necrotrophic life style. Pages 29-50 in: Plant Relationships, 2nd Edition, the Mycota V. H. Deising, ed. Springer-Verlag, Berlin.

van Doorn, W. G., and Woltering, E. J. 2004. Senescence and programmed cell death: Substance or semantics? J. Exp. Bot. 55:2147-2153.

Vicedo, B., de la O Leyva, M., Flors, V., Finiti, I., Del Amo, G., Walters, D., Real, M. D., Garcia-Agustin, P., and Gonzalez-Bosch, C. 2006. Control of the phytopathogen Botrytis cinerea using adipic acid monoethyl ester. Arch. Microbiol. 184:316-326.

Williamson, B., Tudzynski, B., Tudzynski, P., and van Kan, J. A. 2007. Botrytis cinerea: The cause of grey mould disease. Mol. Plant Pathol. 8:561-580.

Winston, F., Dollard, C., and Ricupero-Hovasse, S. L. 1995. Construction of a set of convenient Saccharomyces cerevisiae strains that are isogenic to S288C. Yeast 11:53-55.

AUTHOR-RECOMMENDED INTERNET RESOURCES

Broad Institute Botrytis cinerea database:

www.broadinstitute.org/annotation/genome/botrytis_cinerea ImageJ software: rsb.info.nih.gov/ij 